\title{
Effects of Pressure and Saturation on \\ Seismic Velocities and Impedance Measurements
}

\author{
James G. Berryman \\ University of California \\ Lawrence Livermore National Laboratory \\ P. O. Box 808 L-200 \\ Livermore, CA 94551-9900
}




\section{DISCLAIMER}

This document was prepared as an account of work sponsored by an agency of the United States Government. Neither the United States Government nor the University of California nor any of their employees, makes any warranty, express or implied, or assumes any legal liability or responsibility for the accuracy, completeness, or usefulness of any information, apparatus, product, or process disclosed, or represents that its use would not infringe privately owned rights. Reference herein to any specific commercial product, process, or service by trade name, trademark, manufacturer, or otherwise, does not necessarily constitute or imply its endorsement, recommendation, or favoring by the United States Government or the University of California. The views and opinions of authors expressed herein do not necessarily state or reflect those of the United States Government or the University of California, and shall not be used for advertising or product endorsement purposes.

This is a preprint of a paper intended for publication in a journal or proceedings. Since changes may be made before publication, this preprint is made available with the understanding that it will not be cited or reproduced without the permission of the author.

This work was performed under the auspices of the United States Department of Energy by the University of California, Lawrence Livermore National Laboratory under contract No. W-7405Eng-48.

This report has been reproduced directly from the best available copy.

Available electronically at http://www.doc.gov/bridge

Available for a processing fee to U.S. Department of Energy

And its contractors in paper from

U.S. Department of Energy

Office of Scientific and Technical Information

P.O. Box 62

Oak Ridge, TN 37831-0062

Telephone: (865) 576-8401

Facsimile: (865) 576-5728

E-mail: reports@adonis.osti.gov

Available for the sale to the public from

U.S. Department of Commerce

National Technical Information Service

5285 Port Royal Road

Springfield, VA 22161

Telephone: (800) 553-6847

Facsimile: (703) 605-6900

E-mail: orders@ntis.fedworld.gov

Online ordering: http://www.ntis.gov/ordering.htm

OR

Lawrence Livermore National Laboratory

Technical Information Department's Digital Library

http://www.llnl.gov/tid/Library.html 


\section{Abstract}

Two synthetic data sets were examined to study the possible methods for distinguishing the effects of changes in saturation, and changes in pore pressure, on seismic velocity and impedance measurements. The results show that the results obtained previously on the dependence of laboratory velocity data on changes in saturation carry over without change to the data in these data sets. The assumption in this case is that the only data available are the seismic velocities. This situation can arise in cross-well seismic tomography. Of more direct interest to this project is how these methods and results should change when the data are instead seismic impedance measurments. My main conclusions are that the most appropriate plotting methods for seismic impedance data in order to distinguish changes in saturation, changes in pressure, and saturation changes from pressure changes are $(\rho \mu, \lambda / \mu)$ and $\left(\mu / \lambda, \rho \mu^{2} / \lambda\right)$. All of these plotting coordinates can be computed easily from the impedance data $\rho v_{p}$ and $\rho v_{s}$, since $\rho \mu=\left(\rho v_{s}\right)^{2}$, and $\rho \lambda=\left(\rho v_{p}\right)^{2}-2\left(\rho v_{s}\right)^{2}$, while $\lambda / \mu=\rho \lambda / \rho \mu$ and $\rho \mu^{2} / \lambda=(\rho \mu)^{2} / \rho \lambda$. These choices are not the only possibilities, but they seem to give the desired results in many situations. Some issues remain concerning the details at high porosities and small pore pressures, and these will need to be addressed in future work.

\section{Introduction}

In earlier work, I have shown that effects of changes in saturation on seismic velocity can be understood both qualitatively and quantitatively by considering plots of the data in the planes $(\rho / \mu, \lambda / \mu)$ and $(\rho / \lambda, \mu / \lambda)$. I call these two types of plots the saturation-proxy plot and the data-sorting plot, respectively [1,2]. I did not consider changes in pressure, as there were not nearly so many laboratory data sets for this case as there were for the saturation changes.

The main purpose of the work presented here was: (1) to confirm that the previous approach still applies to reservoir simulation data, and then (2) to proceed to find replacement plotting methods that will work when the data are collected in reflection surveys and therefore will generally be seismic impedance, rather than seismic velocity. An important additional complication has been the introduction of pore-pressure changes that might mask or imitate changes in saturation.

Two data sets were considered. The bulk of the report will address these two data sets in turn. Then I summarize my conclusions.

\section{First Synthetic Data Set}

I received 22 logs for a 20 layer synthetic medium. The logs included one for layer porosity, and three (to mimic the behavior that might be seen in three time-lapse measurements) for various other (seven) properties of the layers. Both saturation and pressure are changing; and the idea is to find a clear signature for each type of change (saturation or pressure) that can be used to distinguish them in real seismic data.

\subsection{Data set}

The seven types of logs changing in time are: 
- water saturation $\left(S\right.$ or $\left.S_{w}\right)$

- oil saturation $\left(S_{0}\right)$

- gas saturation $\left(S_{g}\right)$

- pore pressure $(P)$

- P-velocity $\left(v_{p}\right)$

- S-velocity $\left(v_{s}\right)$

- density $(\rho)$

The main idea I have been pursuing is based on ideas developed previously in two papers, referenced below $[1,2]$.

The ideas basically make use of Gassmann's equations [3] for fluid substitution, together with the fact that density $(\rho)$ changes in a very predictable way with fluid saturation while shear modulus $(\mu)$ is usually constant, when viewed as a function of saturation changes only (except possibly at very low saturations due to chemical effects). These ideas can be used either for velocity data alone (which might be relevant to cross-well seismic or well-log data analysis), or for seismic impedance data (i.t., $\rho v_{p}$ and $\rho v_{s}$, which are most likely to be the data available in surface reflection surveys). These ideas have been very carefully and extensively tested on laboratory data for the "velocity only" case. Some suggestions arising from that work have been made as to whether the extension to seismic impedance data should work, or not, and what form it should take if it does work. The main focus of the new part of this research is therefore to understand issues not previously understood mostly for the impedance data case, but also to some extent for the well-log case.

The data were organized for this study so that a sort of visual time-lapse analysis could be done with them by displaying the data on a variety of correlation plots. Data from the same layer are connected by lines on these plots. It is best to look at the plots in color because there is also color coding (into 5 groups each having 4 contiguous layers), which helps to interpret the results.

The short version of the results obtained is this: The results for velocity data seem to be in complete agreement with our earlier papers on this topic. The early results for the impedance data were not quite as definitive, but some of the examples clearly did show a sorting behavior comparable to that found for the velocity data alone. The problem that seems to arise for the present data sets is that, whereas the shear modulus $\mu$ is essentially independent of the saturation as predicted by Gassmann's analysis, the shear modulus is nevertheless a much stronger function of pressure (at least in these synthetic data) than I might have expected. This fact tended to make the interpretations more difficult in the early stages of this analysis, but eventually led us to a better understanding of what should become the saturation-proxy plot for impedance data.

\subsection{Discussion of various correlation plots}

The coding for the groups of layers from top to bottom are: red (1-4), green (5-8), blue (9-12), black (13-16), and magenta (17-20). Most of the saturation changes are occurring in the 4 middle layers (9-12, blue), while the pressure changes are present in all layers. 


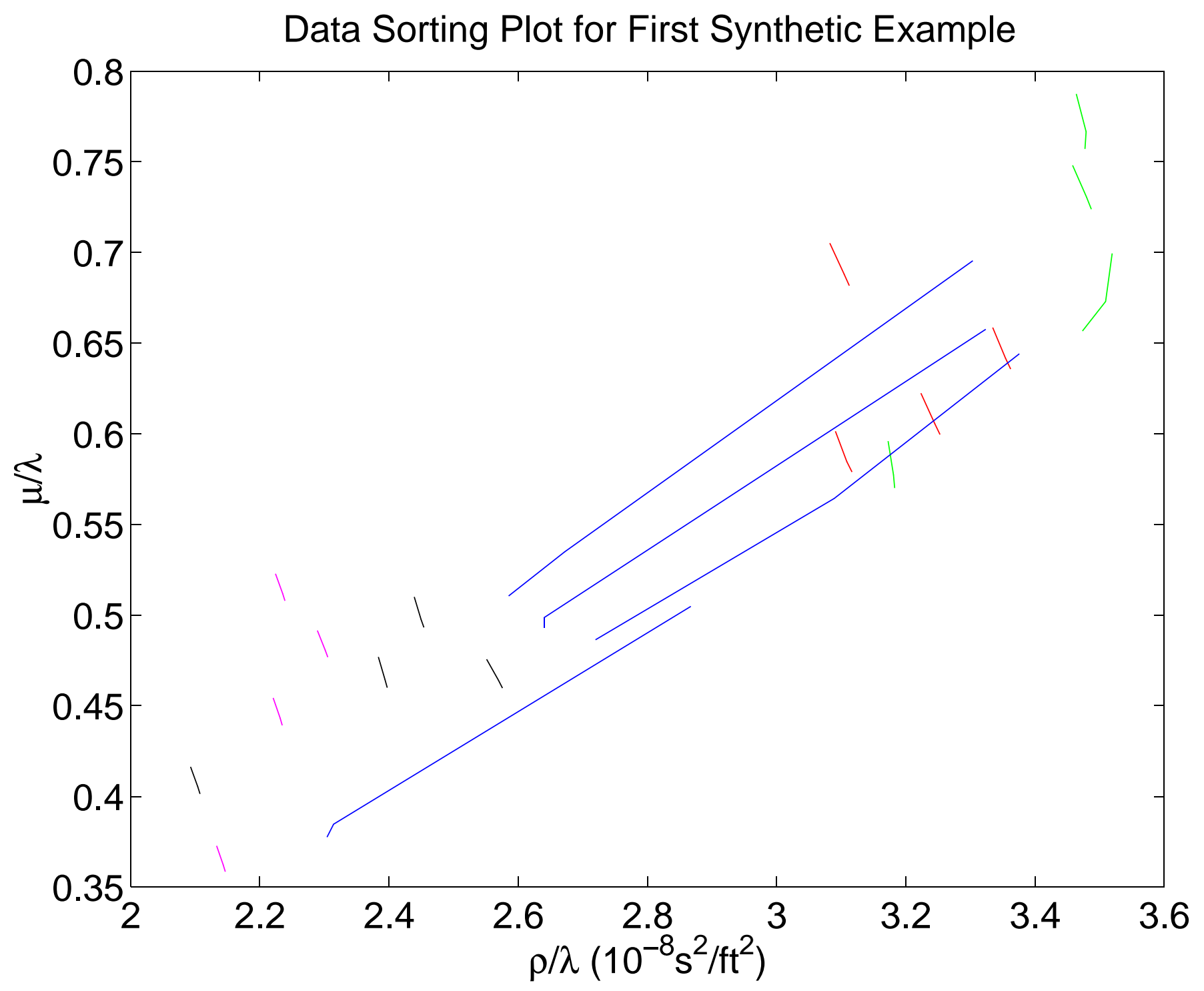

Figure 1: Data-sorting plot. The middle four layers (blue) for which the saturation is changing most dramatically show a very clear linear dependence on this plot, pointed more or less at the origin as expected. 


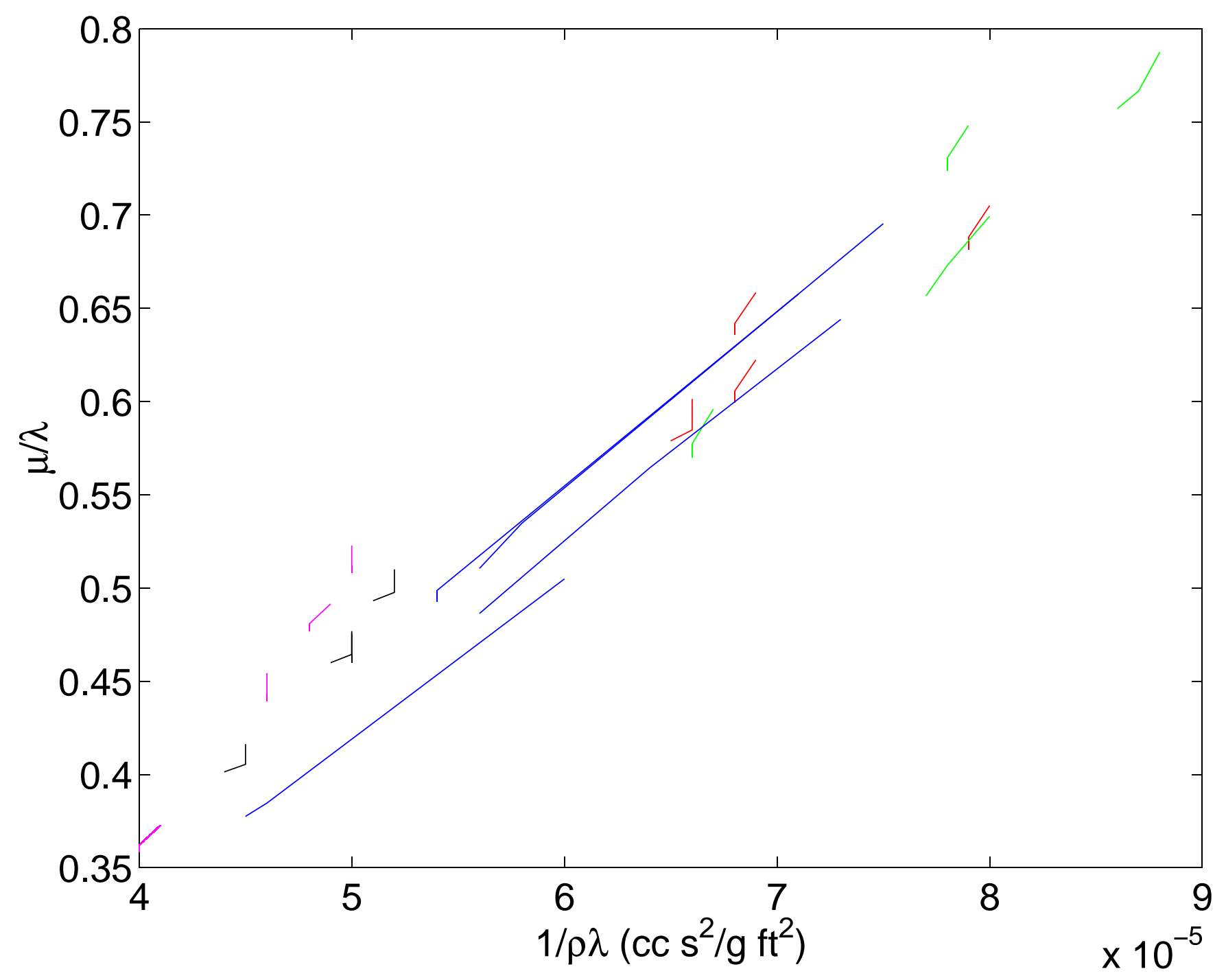

Figure 2: A data-sorting plot for impedance data. The strong linear features of the four middle layers (blue) due to saturation changes are repeated from Figure 1. But the changes in the other layers, although generally much smaller, are not always orthogonal to those of the saturation changes. Compare Figures 5 and 12. 
1. $(\rho / \lambda, \mu / \lambda)$ This plot seen in Figure 1 is called the "data-sorting plot" in the references $[1,2]$; and gives a very clear example of the usefulness of the technique for the data here. The middle four layers for which the saturation is changing most dramatically show a very clear linear dependence on this plot, pointed more or less at the origin as expected. All the remaining layers show much smaller changes and they are all essentially perpendicular to the changes in the middle layers. So clearly the pressure and saturation effects are well separated in this type of plot.

2. $(1 / \rho \lambda, \mu / \lambda)$ This plot seen in Figure 2 is an impedance data version of the previous one. The results are similar but not as definitive. The strong linear features of the four middle layers due to saturation changes are repeated. But the changes in the other layers, although generally much smaller, are not always orthogonal to those of the saturation changes. There is information here, but more care is required in interpretation than for the previous case. Also compare Figures 5 and 12.

3. $(S, \lambda / \mu)$ In Figure 3, the four middle layers (blue) display almost all of the saturation dependence seen in this plot. All the other layers basically have constant saturation and changes occur vertically, apparently because of the changes in the pressure.

4. $(\rho / \mu, \lambda / \mu)$ Figure 4 is the "saturation-proxy plot" motivated in references [1,2]. All the layers show linear trends in essentially the same direction, but the four middle layers show the most dramatic changes. This behavior is not quite what was anticipated from earlier work because of the fairly substantial changes in shear modulus with pressure. But again there is useful information here. Compare the behavior of $(1 / \mu$, const $\times 1 / \mu)$.

5. $(\rho \mu, \lambda / \mu)$ The plot of Figure 5 was expected to be the impedance data equivalent of the preceding one. However, the trends are not at all what was expected. The behavior in truth is very similar to the preceding one, but the trends are all opposite. This could be turned around (if desired by replacing $\rho \mu$ by $1 / \rho \mu$, which is what I initially recommended doing, but I now feel that this figure is fine as is because the interpretations are actually clear now). Again there is useful information here. Pressure and saturation effects are almost orthogonal on this plot. As I learned in later work, when the pressure dependence is dominant, we fully expect the behavior of $(\mu$; const $\times 1 / \mu)$ to dominate on this plot. This is exactly what we see here. Also, compare Figures 2 and 12.

6. $(\rho, \lambda / \mu)$ Figure 6 is a plot that could be done with well-log data, assuming that density data are available. The result is that effects of saturation show up as larger features than those due to pressure. The direction of the trends are however about the same for both saturation and pressure.

7. $(P, 1 / \mu)$ Figure 7 was made to clarify why some of the previously observed behavior was contrary to what I had originally anticipated. It shows very clearly that shear modulus $\mu$ is strongly and inversely proportional to pressure. If the pressure dependence were somewhat weaker, then there would be fewer of the unanticipated results in the preceding plots.

8. $(P, \lambda)$ Same reasoning as for Figure 7 , but this time the trends in Figure 8 are not nearly so predictable. Lamé $\lambda$ does not appear to be a simple function of pressure in these 


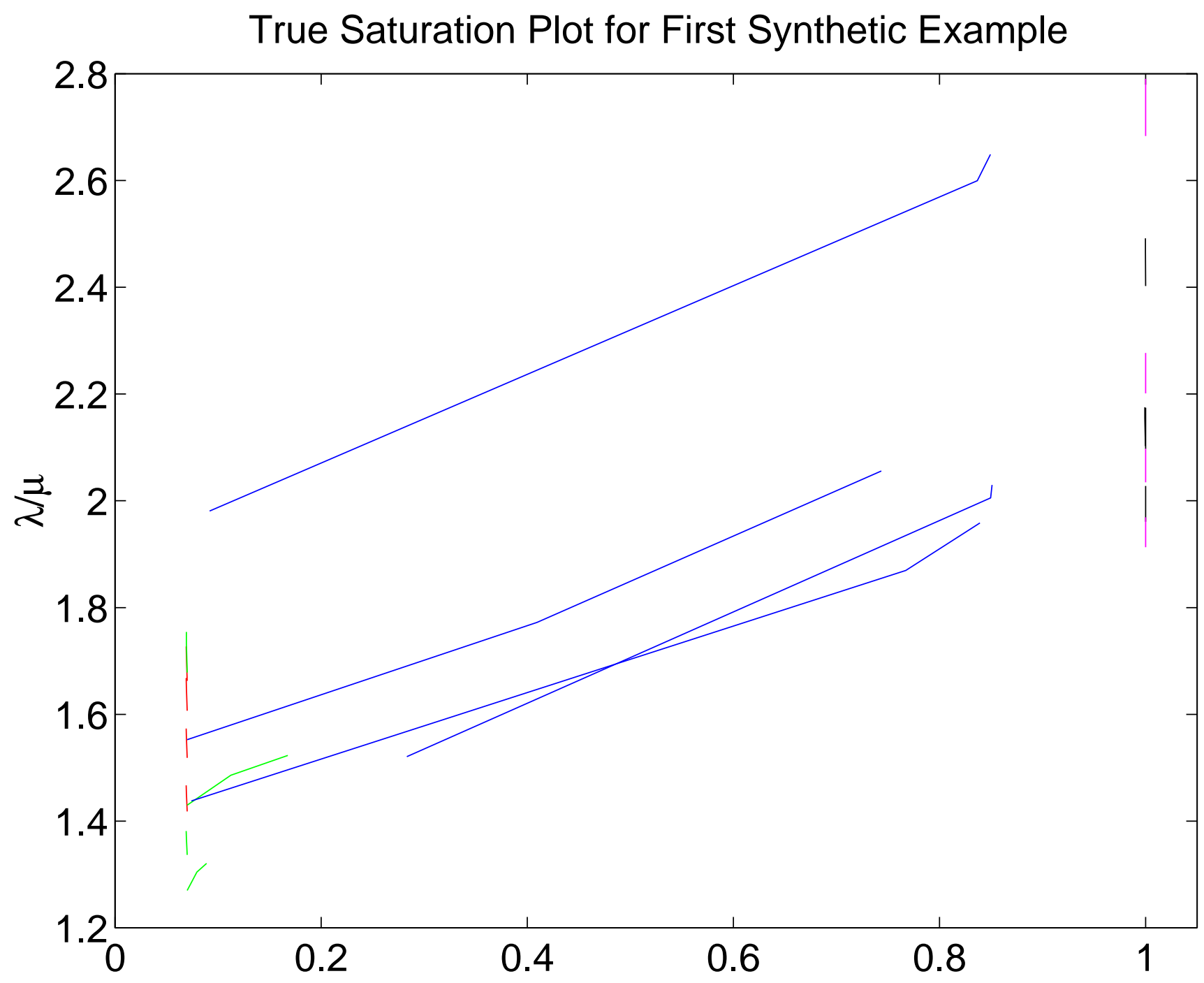

Figure 3: Saturation-data plot. Note that the four middle layers (blue) display almost all of the saturation dependence seen in this plot. 


\section{Saturation Proxy Plot for First Synthetic Example}

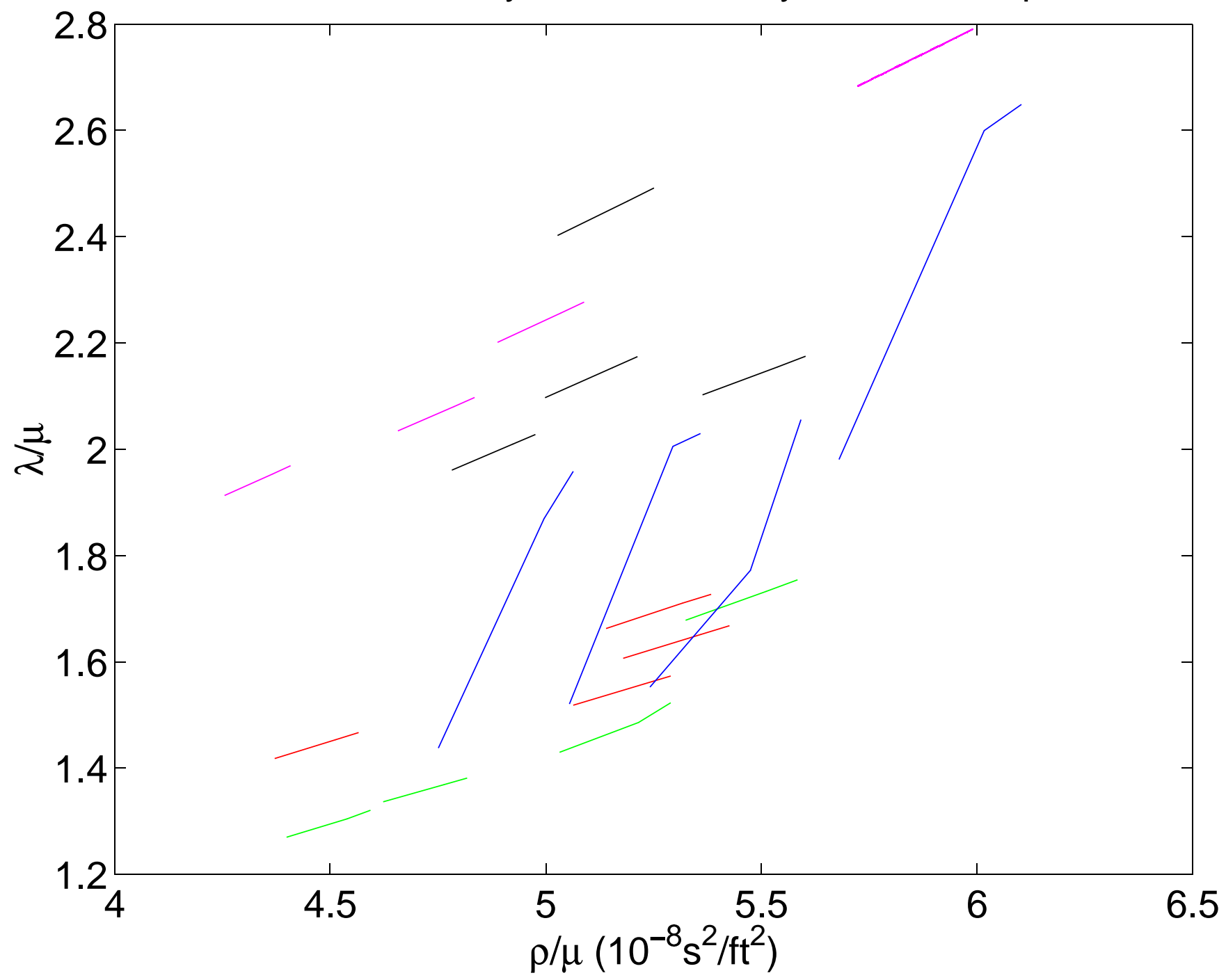

Figure 4: Saturation proxy plot. All the layers show linear trends in essentially the same direction, but the four middle layers (blue) show the most dramatic changes. Compare the behavior of $(1 / \mu$, const $\times 1 / \mu)$. 


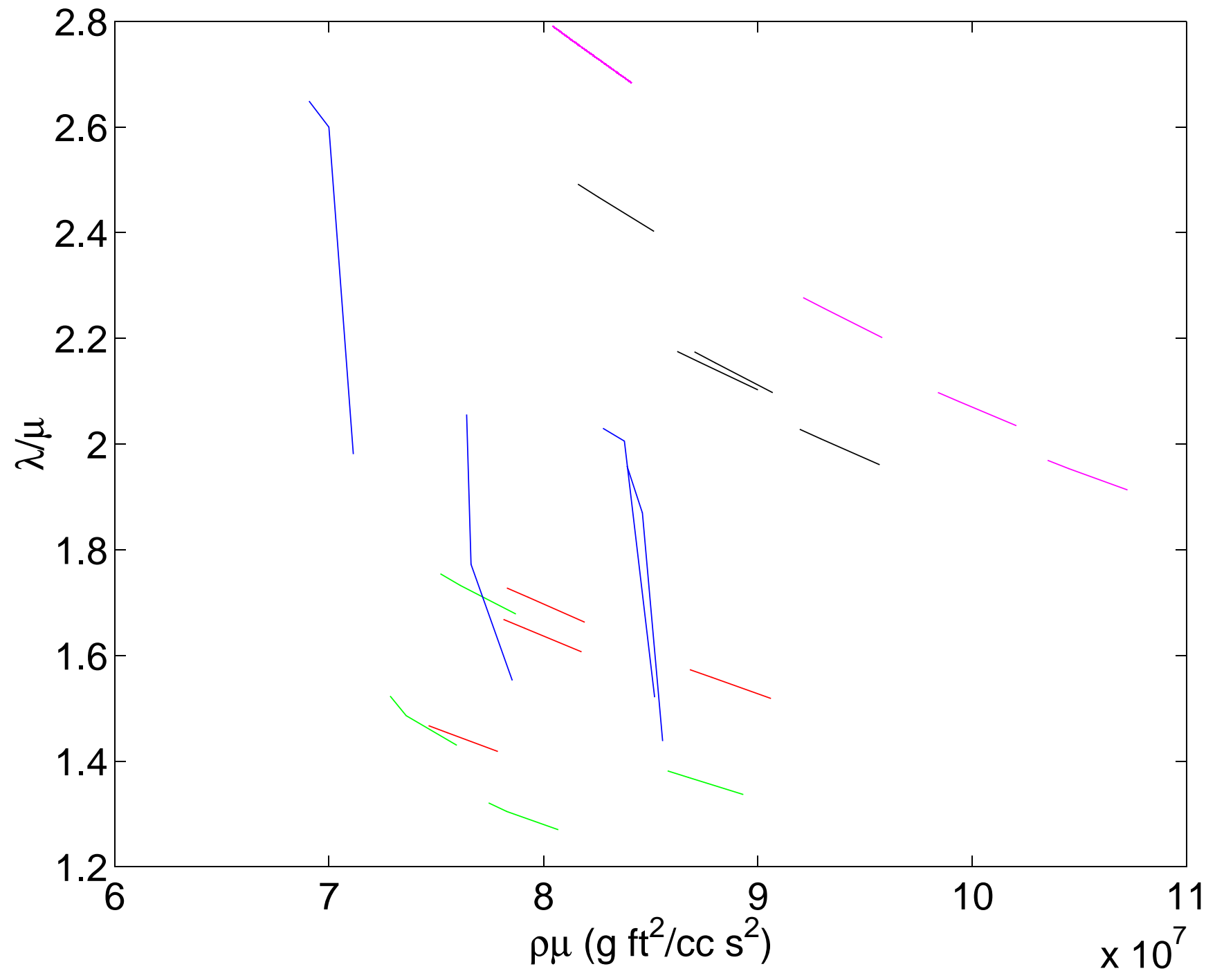

Figure 5: A possible saturation-proxy plot for impedance data. The behavior here is very similar to that in Figure 4, but the trends are all oppositely directed. When the pressure dependence is dominant, we fully expect the behavior of $(\mu$, const $\times 1 / \mu)$ to dominate on this plot. Also, compare Figures 2 and 12. 




Figure 6: Density-proxy plot, a replacement for the saturation-proxy plot when density measurements are available as in well-log data or laboratory data. Effects of saturation show up as larger features than those due to pressure here. 


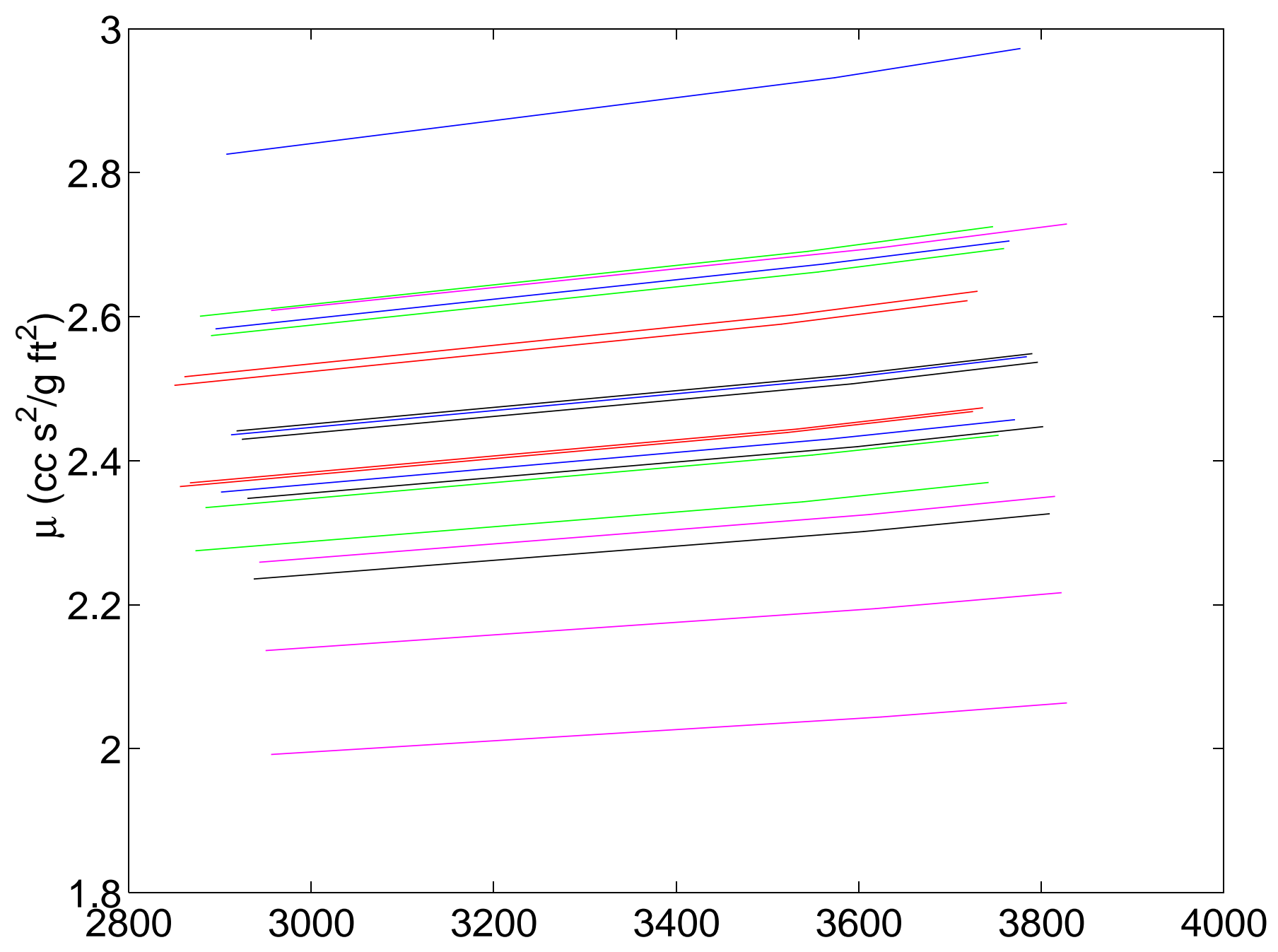

Figure 7: Pore pressure versus inverse of shear modulus. Shear modulus $\mu$ is strongly and inversely proportional to pressure in these simulated data. 


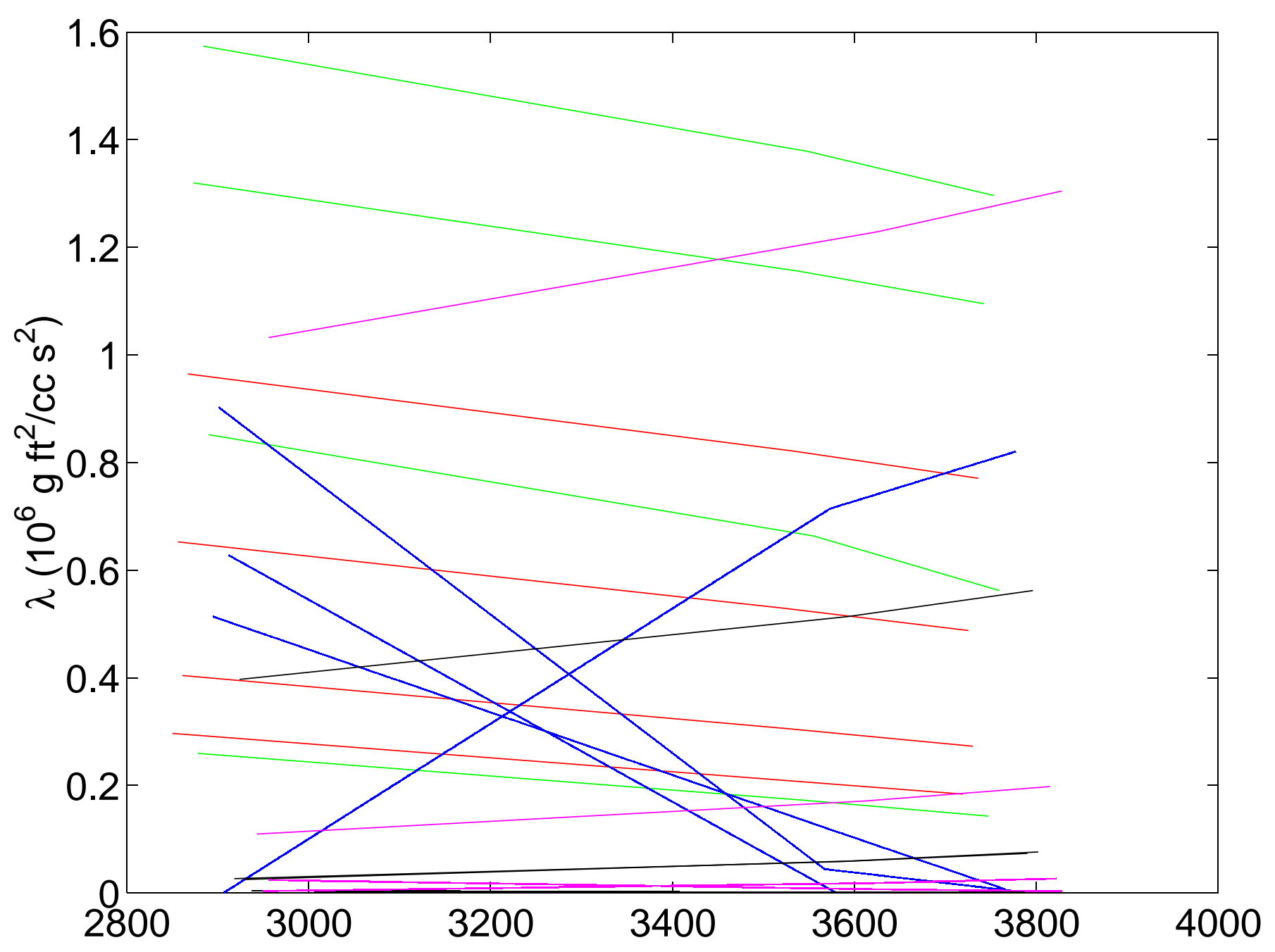

Figure 8: Pore pressure versus Lamé $\lambda$, showing that $\lambda$ is not a simple function of pressure in these synthetic data. 
synthetic data (nor would I expect them to be). To me, the most surprising result here was not this case, but the preceding one.

9. $(\rho, 1 / \mu)$ Figure 9 could be generated with well-log data, assuming that density data are available. Density is constant when saturation does not change, so the distinction between pressure and saturation is clearly visible on this plot.

10. $(\rho, \lambda)$ Another well-log plot, Figure 10 assumes that density data are available. Similar to previous one. But as before the changes in $\lambda$ are not simply correlated with pressure and saturation changes, so these results are not easily interpreted.

11. $(S, \rho)$ Figure 11 shows the predicted simple linear correlation between density and saturation.

12. $\left(\mu / \lambda, \rho \mu^{2} / \lambda\right)$ Figure 12 shows rather simple behavior of a data-sorting plot using only impedance data. This should be compared to Figures 2 and 5 . Also, compare the expected behavior of $(\mu / \lambda$, const $\times \mu / \lambda)$.

\section{Second Synthetic Data Set}

\subsection{General thoughts about impedance data}

After our project meeting on May 9th, I concluded that I had learned several things, two of the most important of which were these:

Issue I. Although the shear modulus is not a function of saturation, it is a fairly strong function of pore pressure. This dependence comes in the form of a gradual decrease in $\mu$ as a function of increasing pore pressure, and the dependence may be approximately of square-root-type, i.t., having a fairly sharp drop at some definite pressure value.

Issue II. The measured data for reflection are not so much absolute values of impedance, as they are relative changes in impedance. It would therefore be helpful to find a means of producing plots that depend only on the relative changes and not on the absolute values.

Conclusions: The square-root-type behavior of the shear modulus should be checked in the data. If the behavior is really exactly a square root, then it would be beneficial to use $\mu^{2}$ in the plots. If the behavior is not exactly square root, but only approximately so then it might be more useful to plot things versus $\ln (\mu)$. Then, if

$$
\mu=\mu_{0}\left(1-F(P) / F\left(P_{0}\right)\right)^{\gamma},
$$

we would have

$$
\ln (\mu)=\mathrm{const}+\gamma \ln \left(1-F(P) / F\left(P_{0}\right)\right) \simeq \text { const }-\gamma F(P) / F\left(P_{0}\right),
$$

which might prove to be a useful plotting tool. In particular, the function $\ln (\mu)$ might be a good proxy for pressure, or some simple function of pressure (such as $P^{2}$, which is what we find later). 


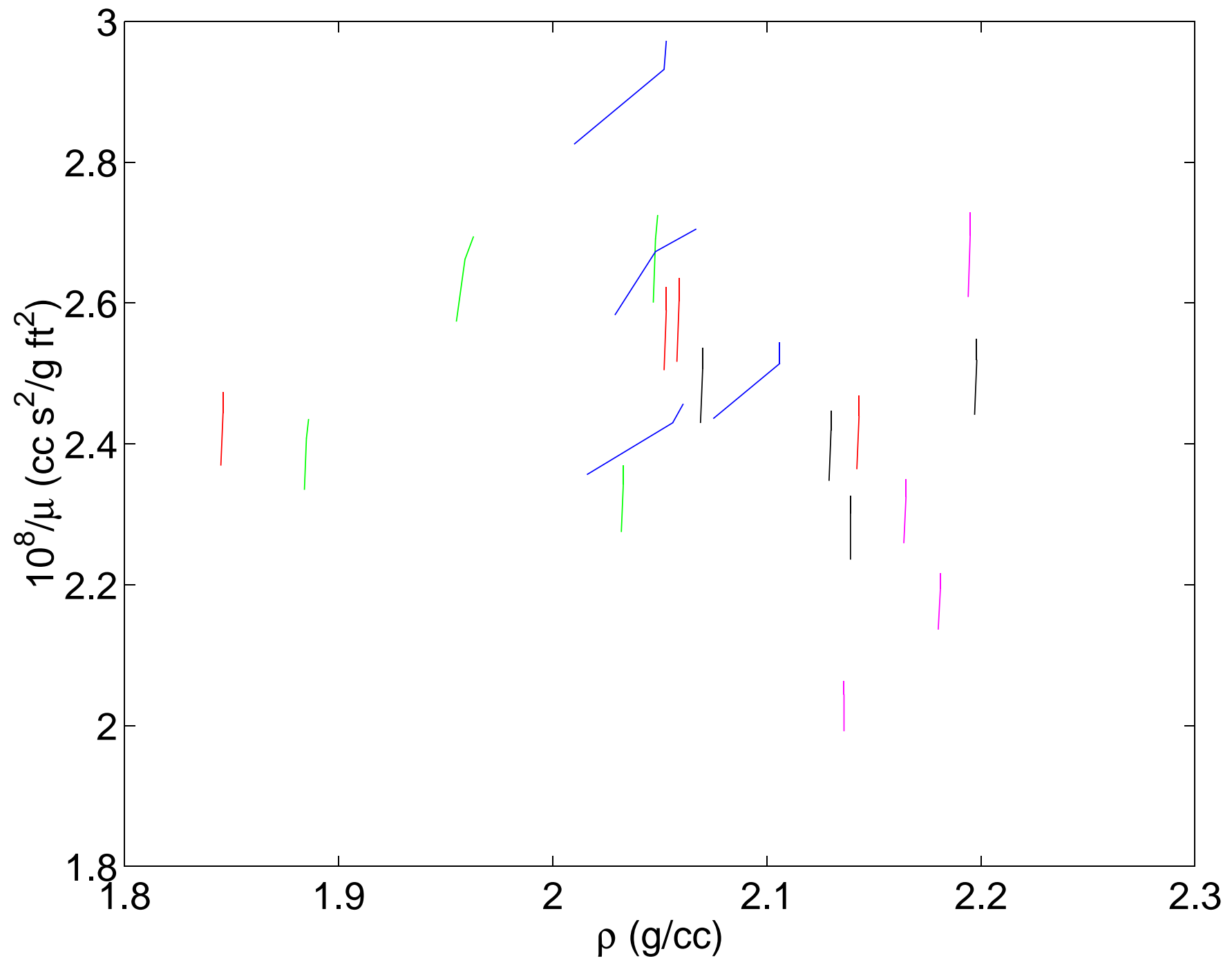

Figure 9: Checking dependence of inverse shear modulus on density. The distinction between pressure and saturation change is clearly visible in this plot. 


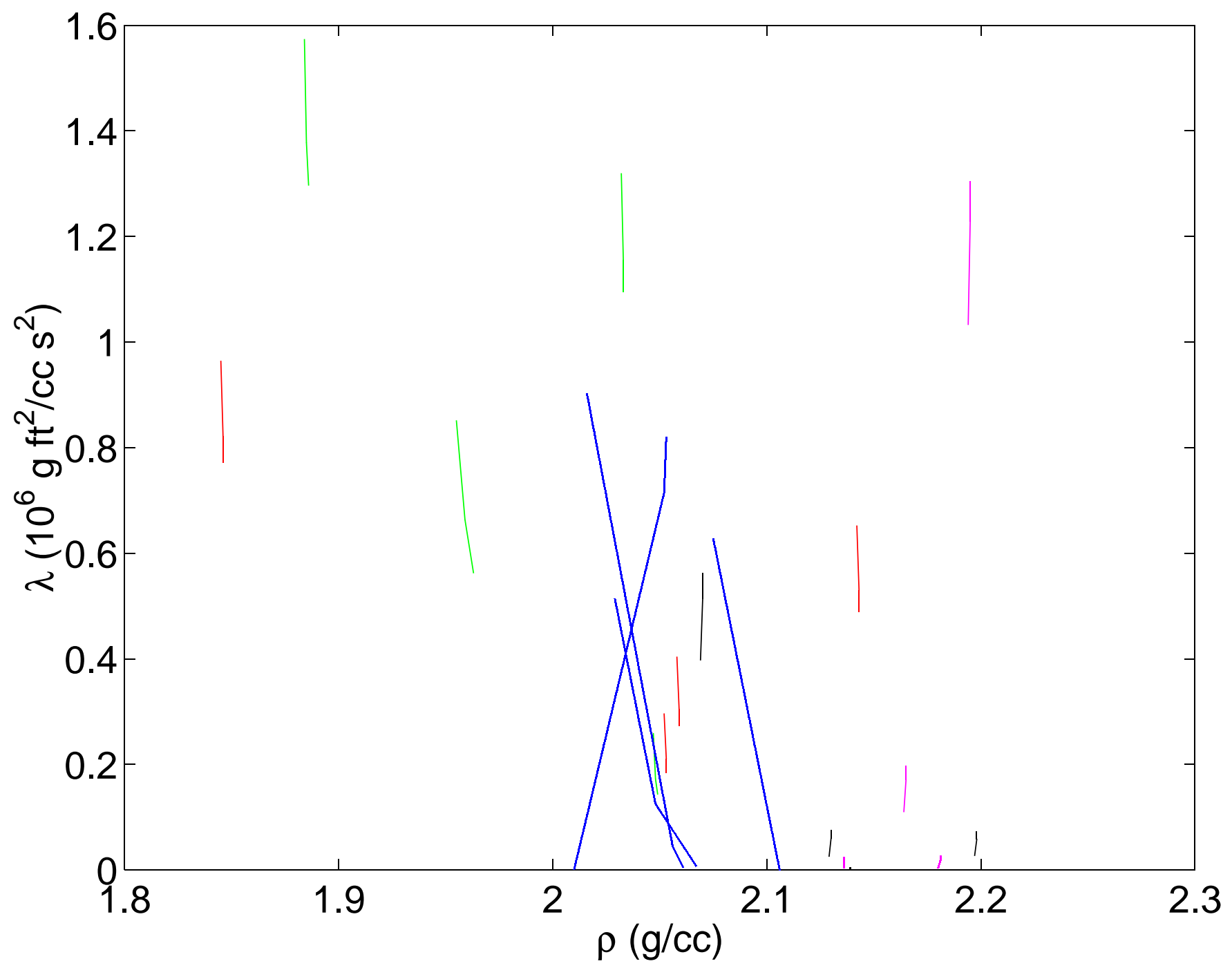

Figure 10: Checking how well $\lambda$ serves as a proxy for density or saturation. The behavior is predictable in the absence of saturation changes, but difficult to predict when both saturation and pressure changes are present. 


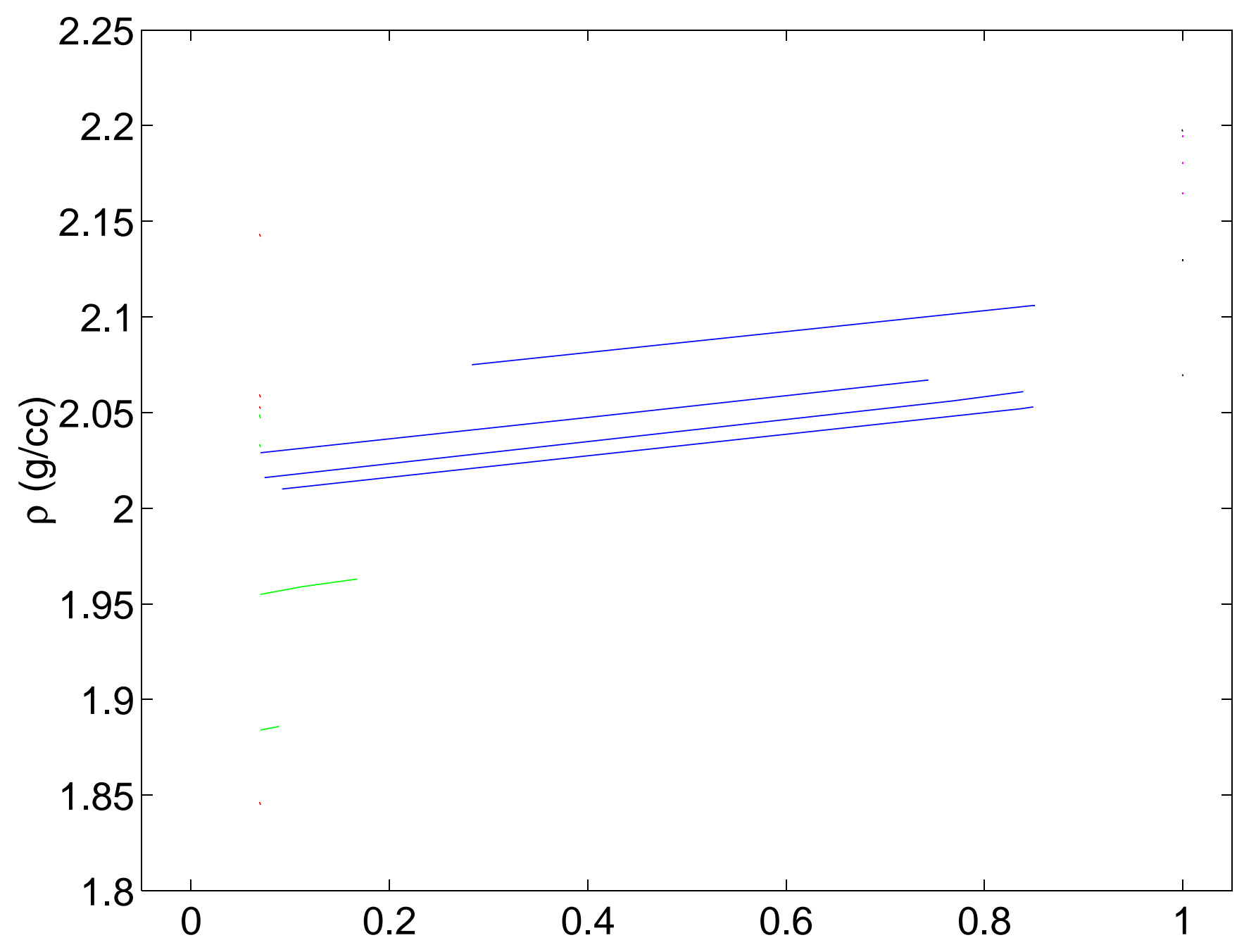

Figure 11: Demonstrating the simple relation (linear) between saturation and density. Good predictability is apparent. 


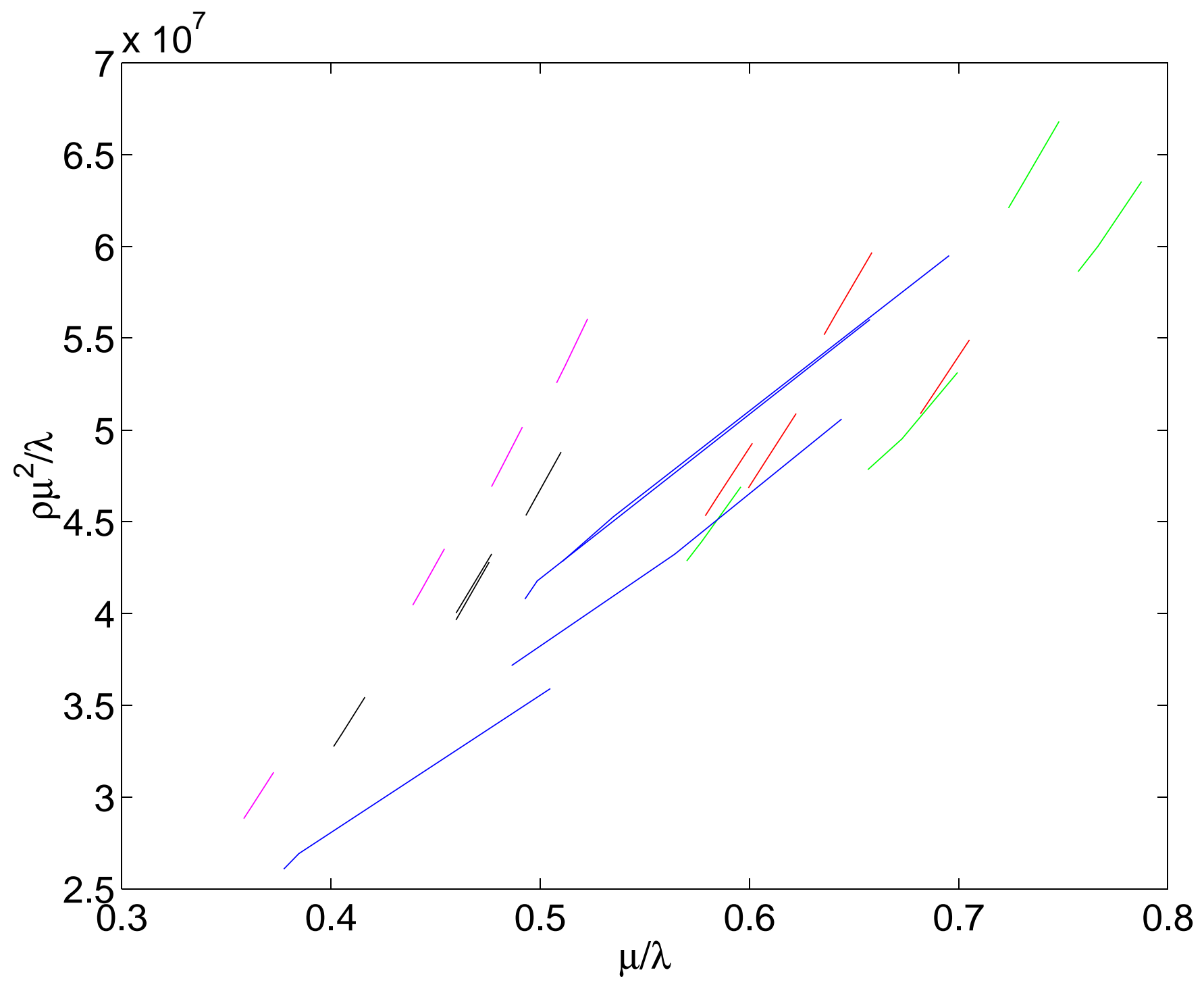

Figure 12: A new data sorting plot for impedance data, showing rather uncomplicated behavior. Compare Figures 2 and 5. Also, compare the expected baviour of $(\mu / \lambda$, const $\times \mu / \lambda)$. 
Similarly, for Issue II, since absolute impedance is not measured directly but must be inferred, it might make sense to consider

$$
\ln \left(\rho v / \rho_{0} v_{0}\right)=\ln \left(1+[\delta(\rho v)] / \rho_{0} v_{0}\right) \simeq[\delta(\rho v)] / \rho_{0} v_{0} .
$$

This idea seems a little less useful than the preceding one, but I thought I should mention it.

\subsection{Data set}

The second data set has:

- 5 porosities

- 11 saturations

- 11 pressures

for a total of $5 \times 11 \times 11=605$ measurements of

- $\rho$

- $v_{p}$

- $v_{s}$

\subsection{Results for $\mu$ and $\lambda$}

To test Issue I above, and for my own understanding of what has gone into this reservoir simulation code, I needed to check how $\mu$ and also $\lambda$ are changing with pressure. I tried several things for $\mu$, and in fact I did not think to do it at first, but later - after seeing the results decided that I definitely needed to test $\lambda$ also.

1. $\left(P^{2}, \ln (\mu)\right)$ Figure 13 , for $S=1$ which is the fully water saturated case, shows clearly that the main dependence on pressure is

$$
\mu=\mu_{0}\left(1-P^{2} / P_{0}^{2}\right)^{\gamma} .
$$

I have not tried to figure out either $P_{0}$ or $\gamma$, as it is a product of these two unknowns $\left(\gamma / P_{0}^{2}\right)$ that determines the slope in this figure. There is a very small region of deviation from this basic behavior at the smallest pressure values.

2. $\left(P^{2}, \ln (\lambda)\right)$ Figure 14 shows that $\lambda$ also has a pressure dependence something like

$$
\lambda=\lambda_{0}\left(1-P^{2} / P_{0}^{2}\right)^{\gamma^{\prime}}
$$

The overall dependence on $P$ is significantly weaker for $\lambda$ than for $\mu$. But, the $\lambda$ behavior is nevertheless more complex, especially at the highest porosities, with stronger deviations at lower pressures. This is important because it shows up very clearly as an anomalous bending of the curve in one of the plots I will discuss later, and in fact that is why I looked at this plot in the first place. I needed to explain (understand) the source of this anomaly. 


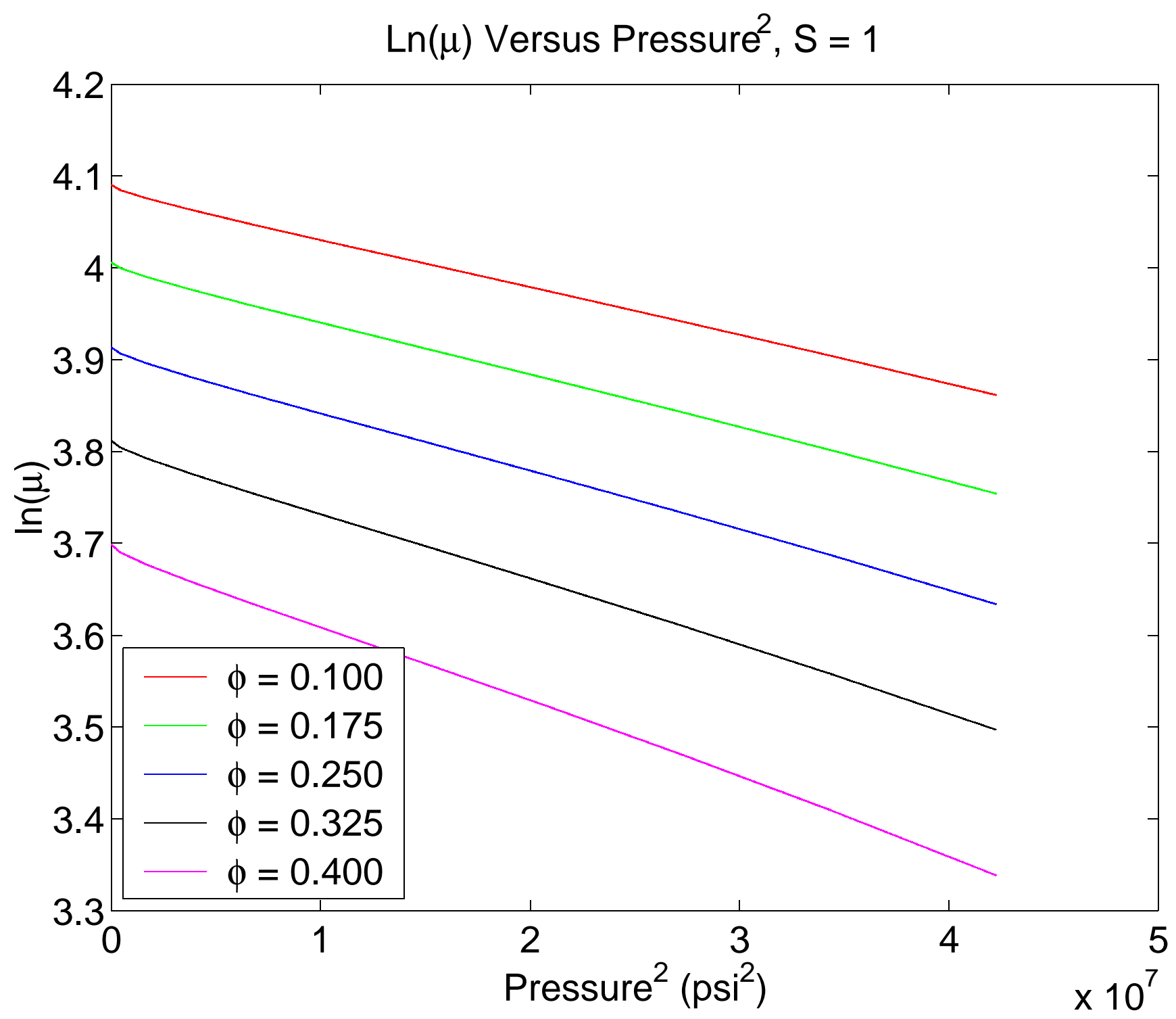

Figure 13: Logarithm of $\mu$ versus pore pressure squared, showing that the main dependence of $\mu$ on pressure is quadratic in pressure. This is for $S=1$, which corresponds to the fully water-saturated case. 


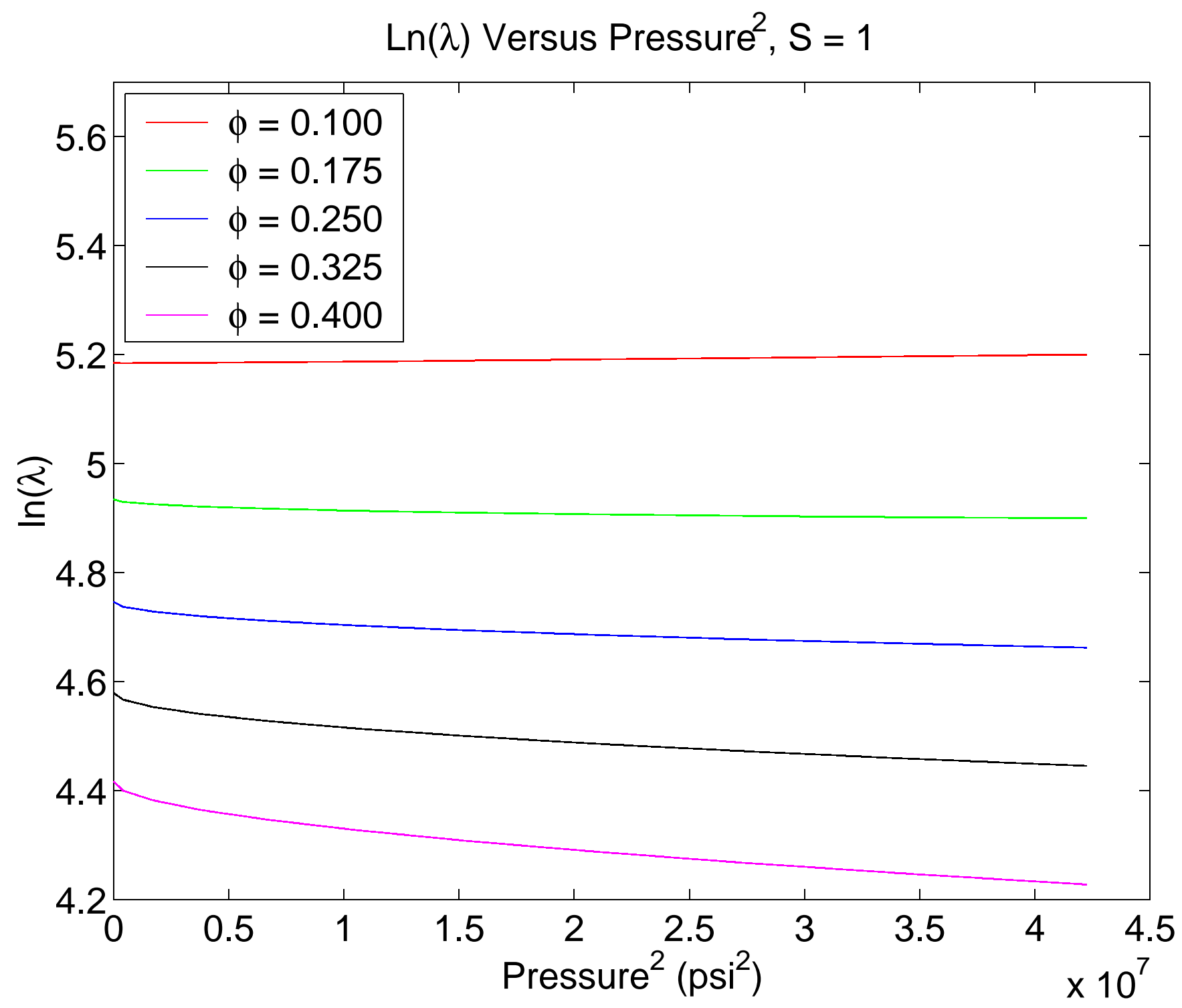

Figure 14: Logarithm of $\lambda$ versus pore pressure squared, showing that the main dependence of $\lambda$ on pressure is similar but not identical to that of $\mu$. The overall dependence on $P$ is significantly weaker for $\lambda$ than for $\mu$, especially at lower porosities. This is for $S=1$, which corresponds to the fully water-saturated case. 


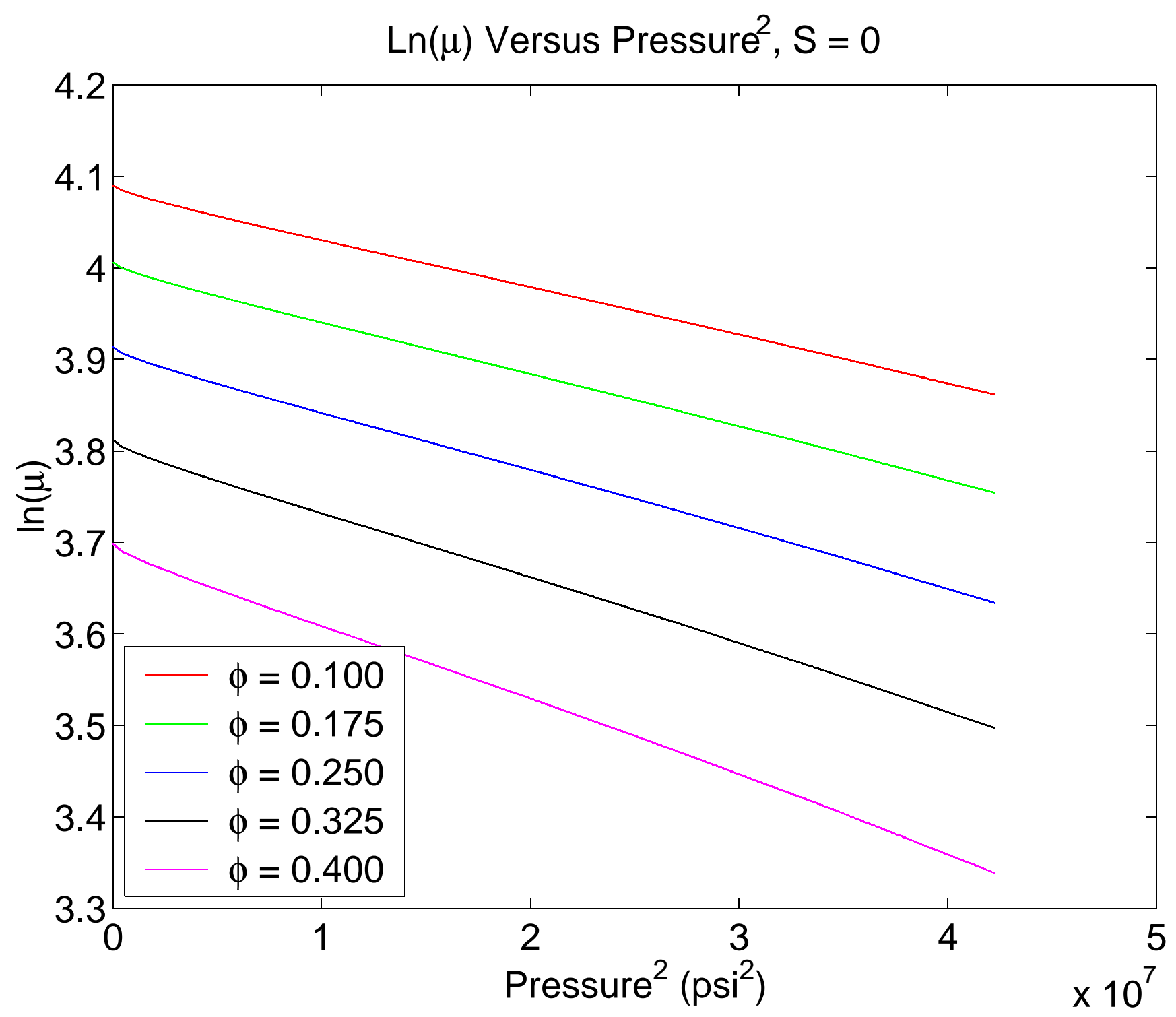

Figure 15: Logarithm of $\mu$ versus pore pressure squared, showing that the main dependence of $\mu$ on pressure is quadratic in pressure. This is for $S=0$, which corresponds to the fully oil-saturated case. This Figure is indistinguishable from Figure 13. 
3. $\left(P^{2}, \ln (\mu)\right)$ Figure 15 shows the same dependence as Figure 13, but for the oil saturated case.

4. $\left(P^{2}, \ln (\lambda)\right)$ Figure 16 , for the fully oil saturated case (compare Figure 14), shows that $\lambda$ is a weaker function of pressure than is $\mu$, but also showing that $\lambda$ does in fact depend on the fluid preperties, unlike $\mu$.

It would be good to understand where the deviations at high porosity and low pressure change are coming from. I have considered issues like Hertzian contacts, and gas coming out of solution, but I think both of those lead to deviations that would have opposite sign to what is observed here. So what is causing it? Is this a real effect, or is it something anomalous that has crept into the code for reasons we do not understand? This issue was tentatively resolved at our second meeting. More will be added concerning the resolution of this issue when we have more data conerning the behavior of the bulk modulus of the fluids as a function of pore pressure in these codes.

\subsection{Results for saturation-proxy and data-sorting plots, especially for impedance only data:}

The short version is this: I tried many things over a period of a couple of days. I finally landed on a combination that I think works about as well for impedance only data, as my previous plots did for velocity only data.

The saturation-proxy plot is:

$$
(\rho \mu, \lambda / \mu)=(\rho \mu,(\rho \lambda / \rho \mu))
$$

This should be compared to $(\rho / \mu, \lambda / \mu)$. When the only thing that varies is saturation, both of these have the same character because $\mu$ is constant. So both plots capture the dependence of $\lambda$ on changes in $\rho$, because $\rho$ is always a very good proxy for saturation.

The data-sorting plot is:

$$
\left(\mu / \lambda, \rho \mu^{2} / \lambda\right)=\left((\rho \mu / \rho \lambda),(\rho \mu)^{2} /(\rho \lambda)\right)
$$

This should be compared to $(\rho / \lambda, \mu / \lambda)$. When the only variation is in saturation, these two are virtually the same except that I have interchanged the abscissa and ordinate. The shear modulus is constant when only saturation changes, and therefore the main feature of these plots in this case is a long streak of data along a positively sloped line that has slope given approximately by $\rho \mu$ in this case, and $\mu / \rho$ in the previous case. Since $\rho$ does not change much due to pressure or small changes in porosity, the $\mu$ dependence (and therefore pressure dependence) dominates the "sorting" characteristic of both of these plots.

Also, note that the two impedance only plots have the straight-line-to-straight-line transform character again, i.e., as we transform from one plane to the other, straight lines are transformed into straight lines. For example,

$$
\rho \mu^{2} / \lambda=A+B \mu / \lambda
$$

implies that

$$
\rho \mu=A \lambda / \mu+B
$$




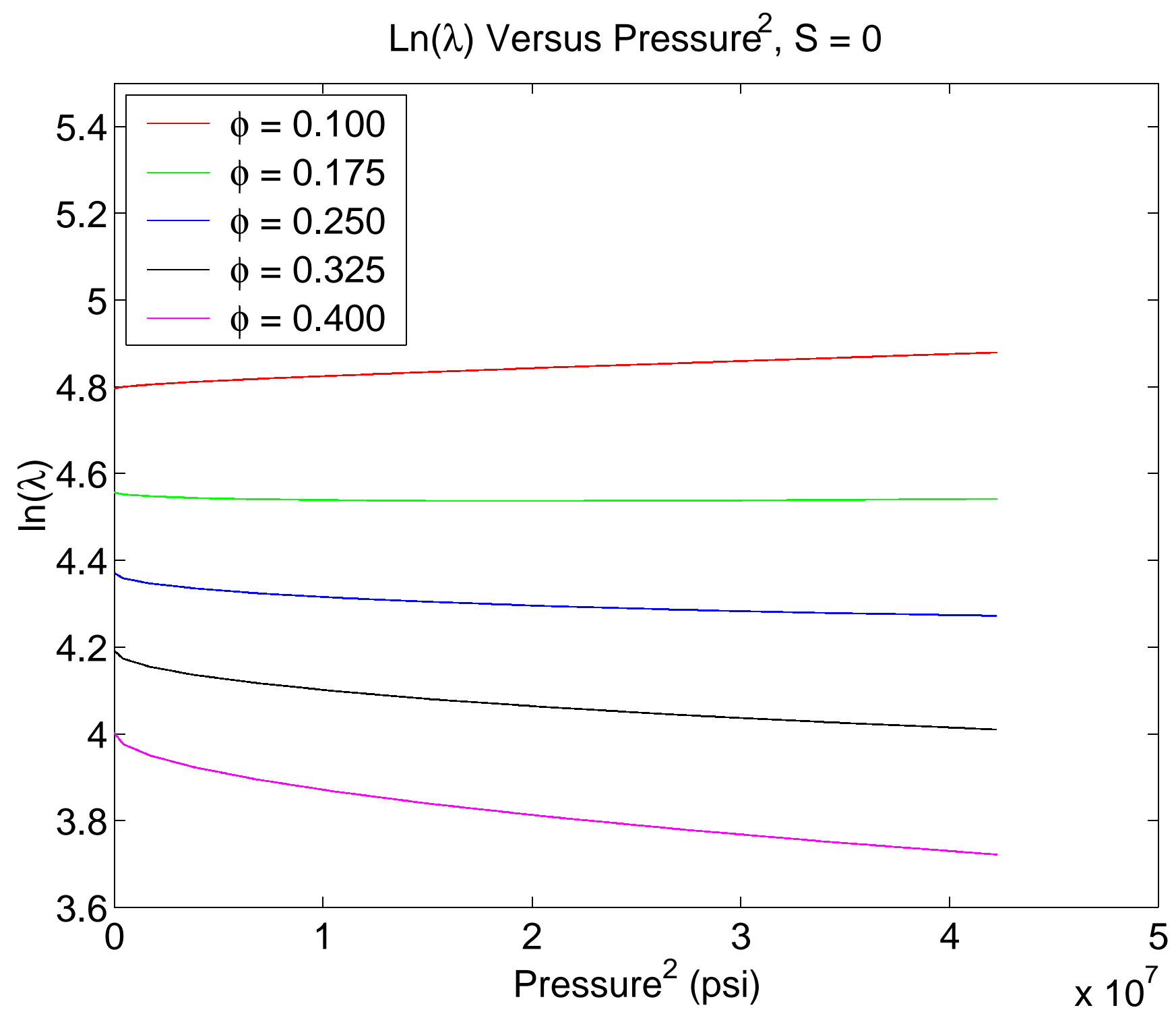

Figure 16: Logarithm of $\lambda$ versus pore pressure squared, showing that the main dependence of $\lambda$ on pressure is similar but not identical to that of $\mu$. The overall dependence on $P$ is significantly weaker for $\lambda$ than for $\mu$, especially at lower porosities. This is for $S=0$, which corresponds to fully oil saturated. This Figure is similar to but distinguishable from Figure 14, showing that the fluid does make a difference to the $\lambda$ dependence. 


$$
\lambda / \mu=-B A^{-1}+A^{-1} \rho \mu .
$$

This property may not be essential, but it helps a lot in interpreting the results of the two plots.

Saturation-proxy plot examples:

1. $(\rho \mu, \lambda / \mu)$ for the two extreme values of pore pressure, 0 and 6500 psia. See Figures 17 and 18.

2. $(\rho \mu, \lambda / \mu)$ for each of the five fixed porosities, $\phi=0.1,0.175,0.25,0.325$, and 0.4 . See Figures 19-23.

In these plots the effects of saturation and pressure are usually easily distinguished. If pressure changes are absent, then the only effect is a rapid increase in $\lambda$ as $\rho$ increases. If pressure is changing at fixed porosity and saturation, then the main effect is like $(\mu$; const $\times 1 / \mu)$; as pressure increases, $\mu$ decreases and we see a curve having mostly negative slope on these plots.

There is some unexpected curvature at the lowest pressures, especially for the highest porosities. This is one place where the issue of dependence of $\lambda$ on pressure is important.

Data-sorting plot examples:

3. $\left(\mu / \lambda, \rho \mu^{2} / \lambda\right)$ for the two extremes of fixed pressure, 0 and 6500 psia. See Figures 24 and 25 .

4. $\left(\mu / \lambda, \rho \mu^{2} / \lambda\right)$ for each of the five fixed porosities, $0.1,0.175,0.25,0.325,0.4$. See Figures $26-30$.

Since most of the rapid changes are occurring in either $\mu$ due to pressure or $\lambda$ due to saturation, this plane has the feature that (almost always) the results line up along straight lines [consider $(\mu / \lambda$, const $\times \mu / \lambda)]$; and the slopes of the lines are well approximated by $\rho \mu$. Shear modulus $\mu$ might change because of changes in porosity (Figures 24 and 25) or because of changes in pressure (Figures 26-30). There is actually some observable separation due to changes in $\rho$ with saturation in Figures 26-30.

The most startling feature of these plots is at the highest porosities and the lowest pressures. We see strong hooks in the curves. This can only be caused by having $\lambda$ changing more rapidly than $\mu$ but not quite as rapidly as $\mu^{2}$. The result is some very strong curvature at the lowest pressures, and especially so for the highest porosities. This is the place where the issue of dependence of $\lambda$ on pressure discussed above is most important. If this is a real effect, it could be useful, and - if not - it is just an annoyance for this analysis.

Besides the plots mentioned explicitly here, I generated a number of other plots to test other hypotheses. I concluded that the rest were not as useful as the ones mentioned here explicitly.

Also, the codes I used to generate all the plots are available. Everything was designed for easy plotting using Matlab. 


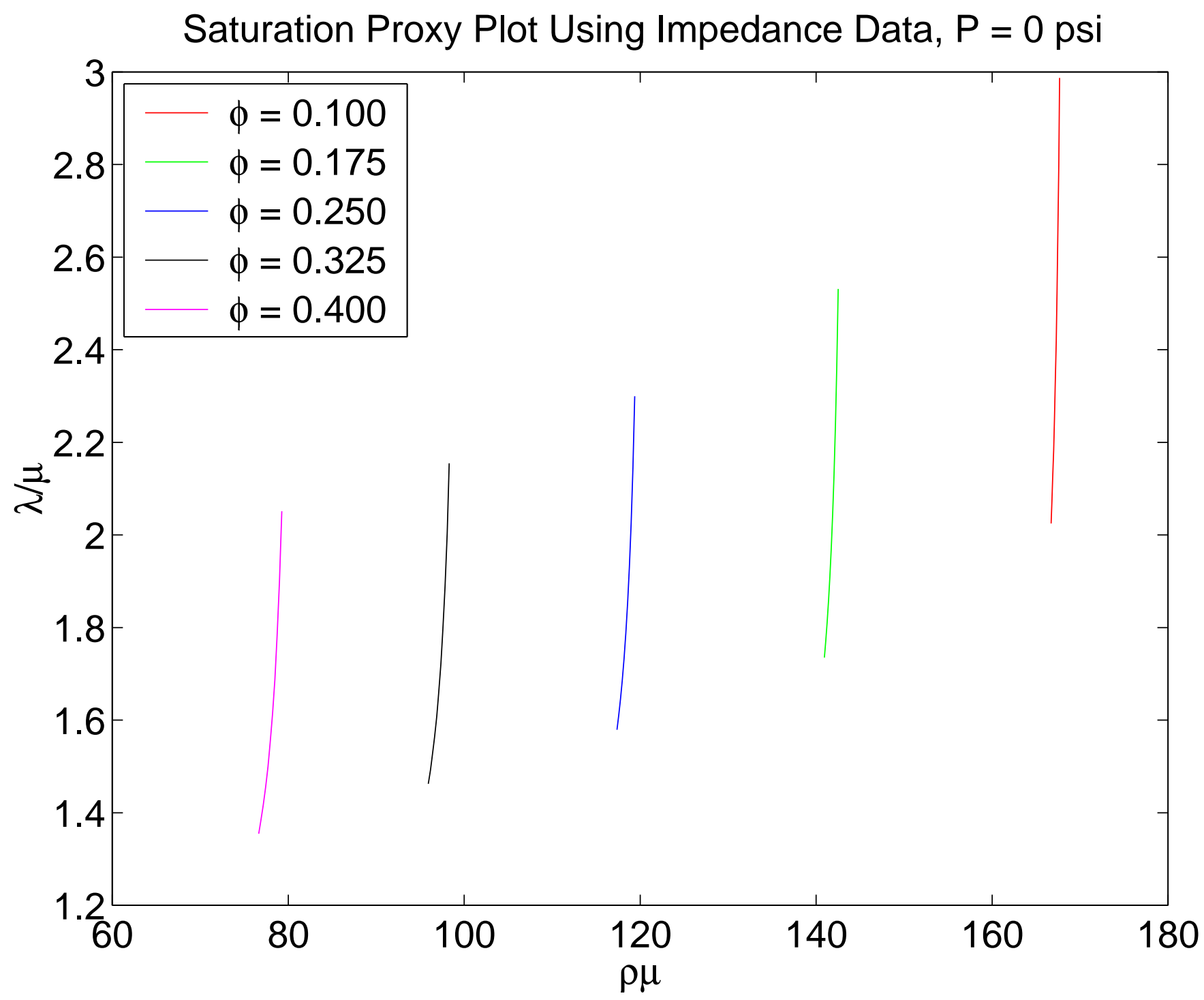

Figure 17: Pressure $=0$ psia. Lamé $\lambda$ changes rapidly with small changes in density. This plot is also useful as a data-sorting plot, as the different values of porosity are very well separated. 


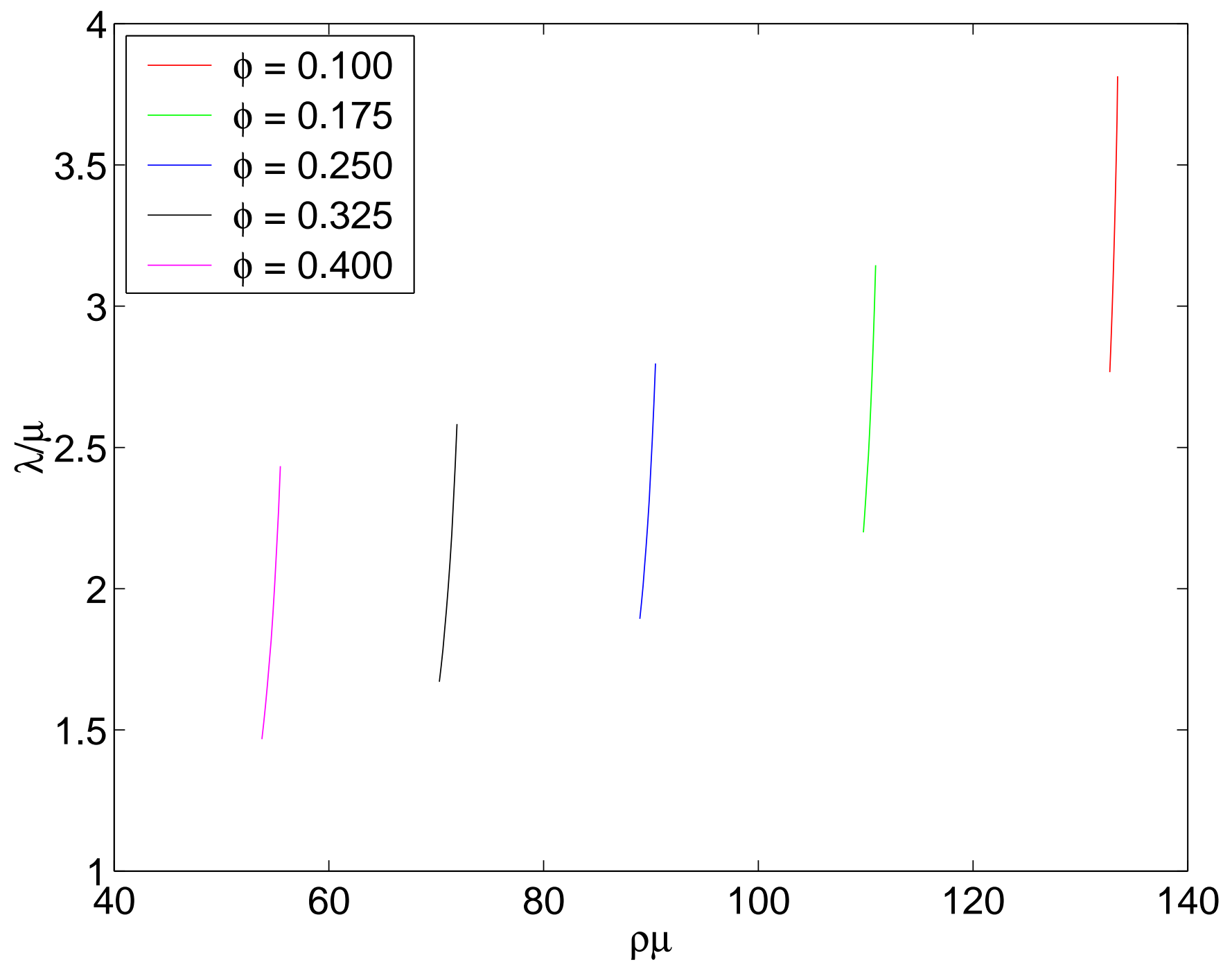

Figure 18: Pressure $=6500$ psia. Lamé $\lambda$ changes rapidly with small changes in density. This plot is also useful as a data-sorting plot, as the different values of porosity are very well separated. 


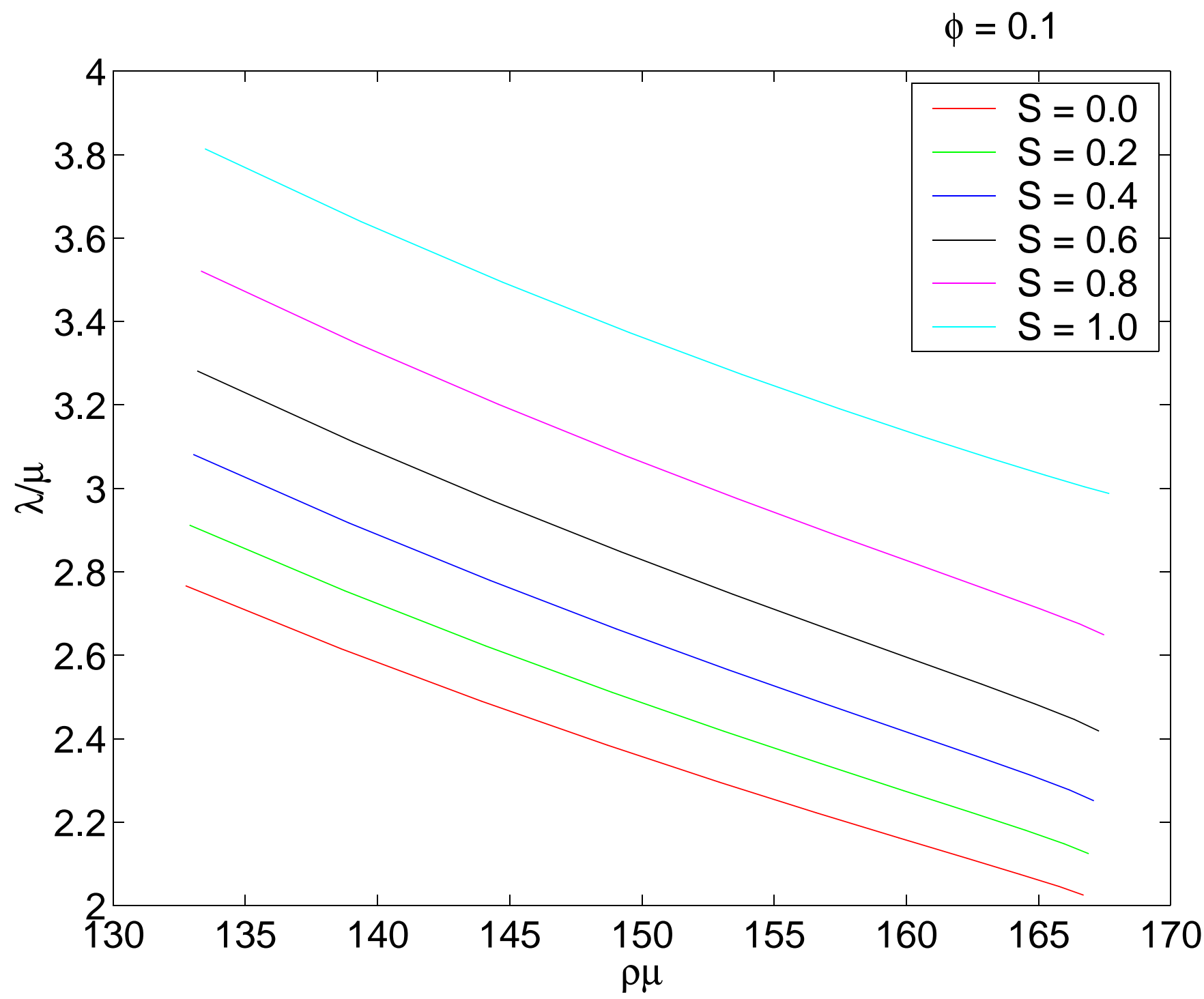

Figure 19: Porosity $\phi=0.1$. Shows the expected inverse correlation with changes of pressure. Compare the expected behavior of $(\mu$; const $\times 1 / \mu)$. 


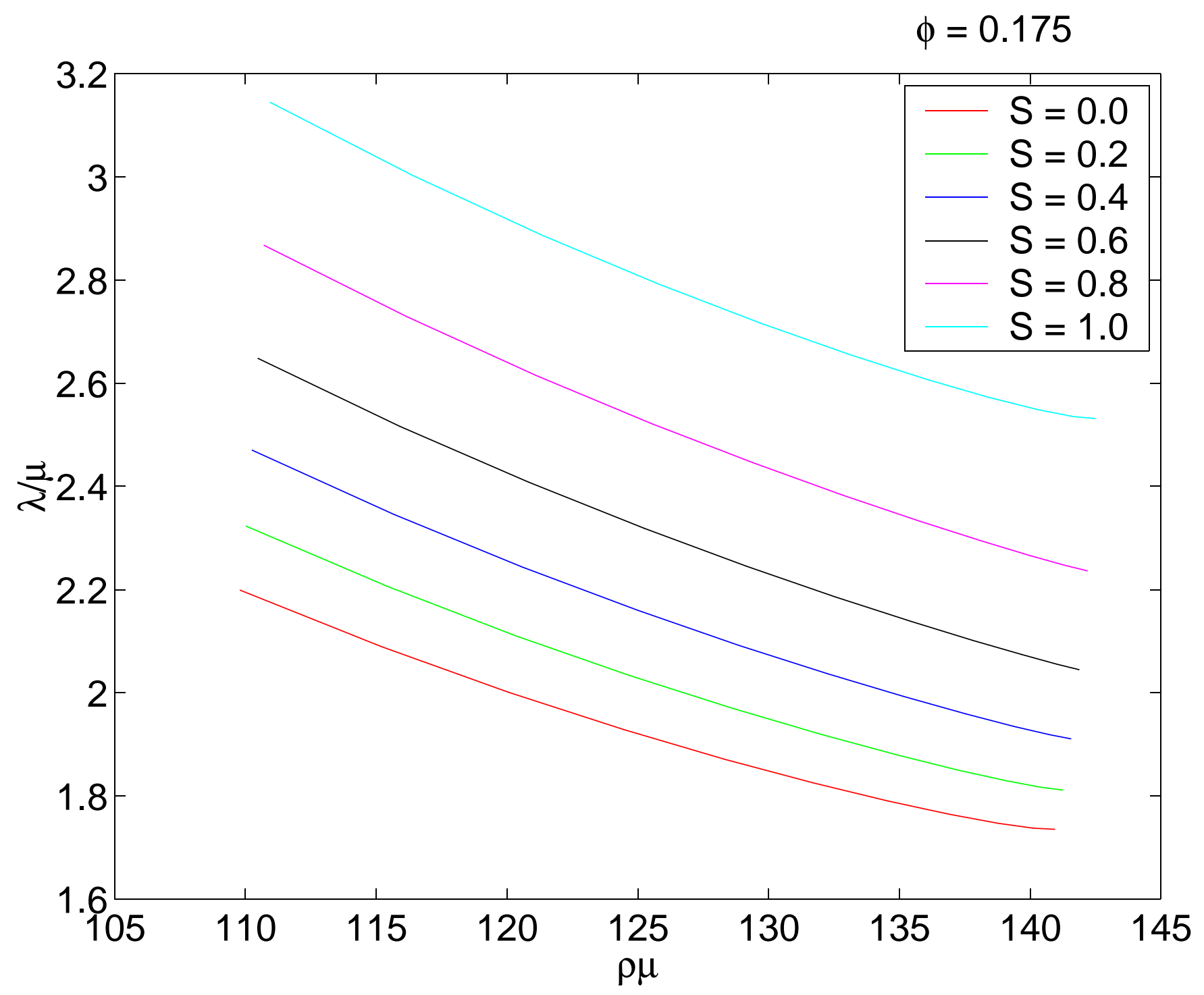

Figure 20: Porosity $\phi=0.175$. Shows the expected inverse correlation with changes of pressure. Compare the expected behavior of $(\mu$; const $\times 1 / \mu)$. 


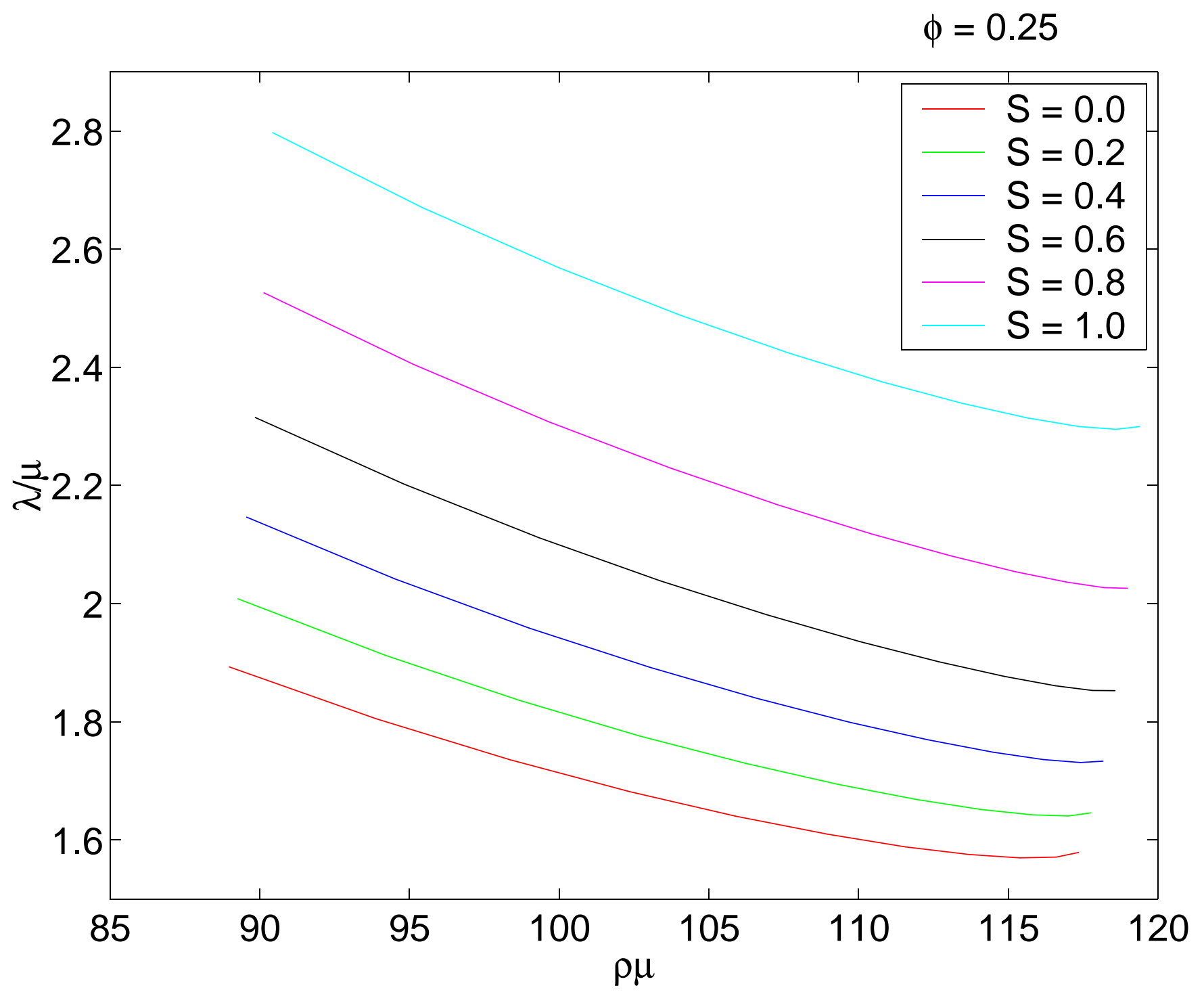

Figure 21: Porosity $\phi=0.25$. Shows the expected inverse correlation with changes of pressure. Compare the expected behavior of $(\mu$; const $\times 1 / \mu)$. 


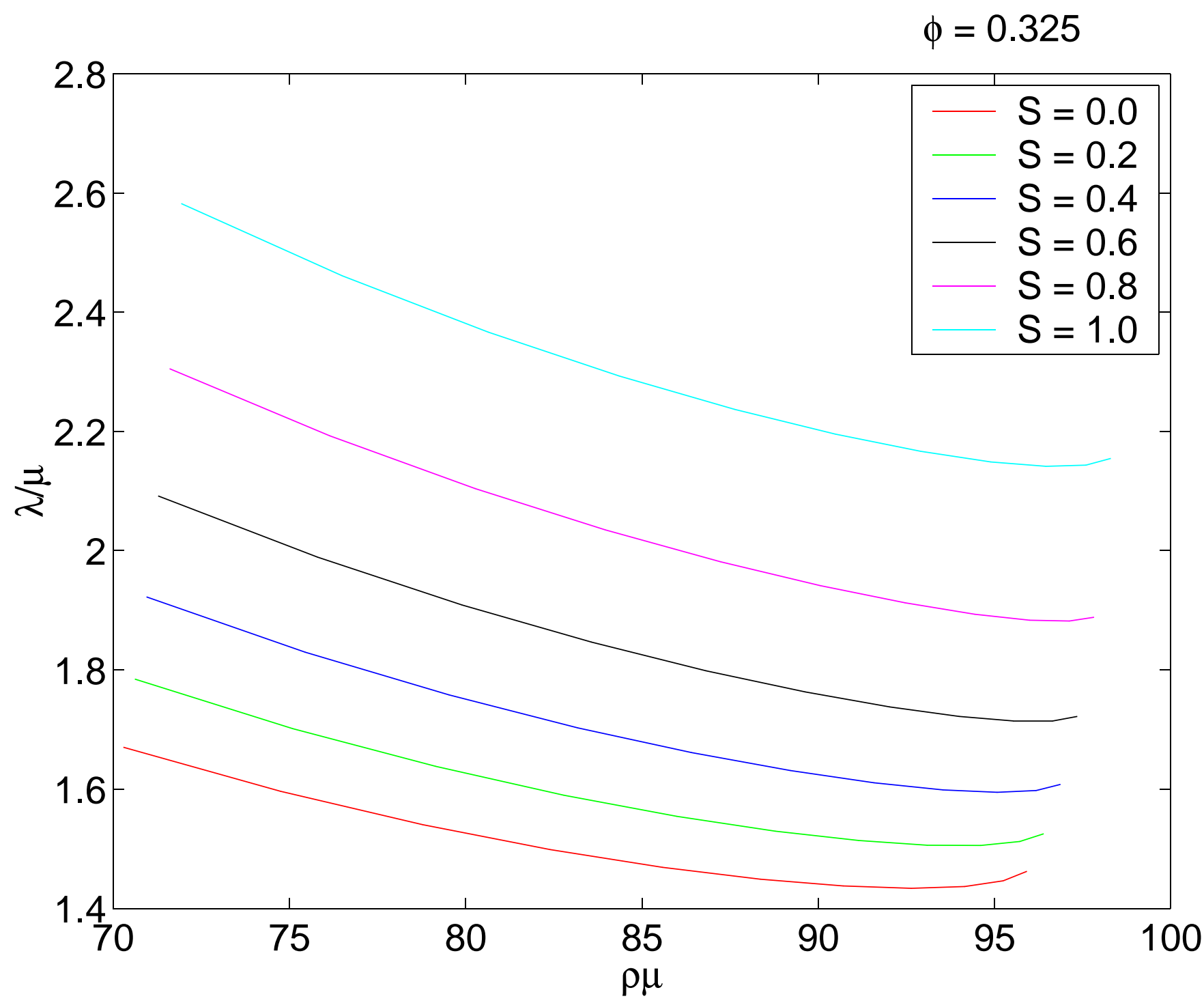

Figure 22: Porosity $\phi=0.325$. Shows the expected inverse correlation with changes of pressure, but some deviations from simple behavior at the lower porosities are observed. Compare the expected behavior of $(\mu$, const $\times 1 / \mu)$. 


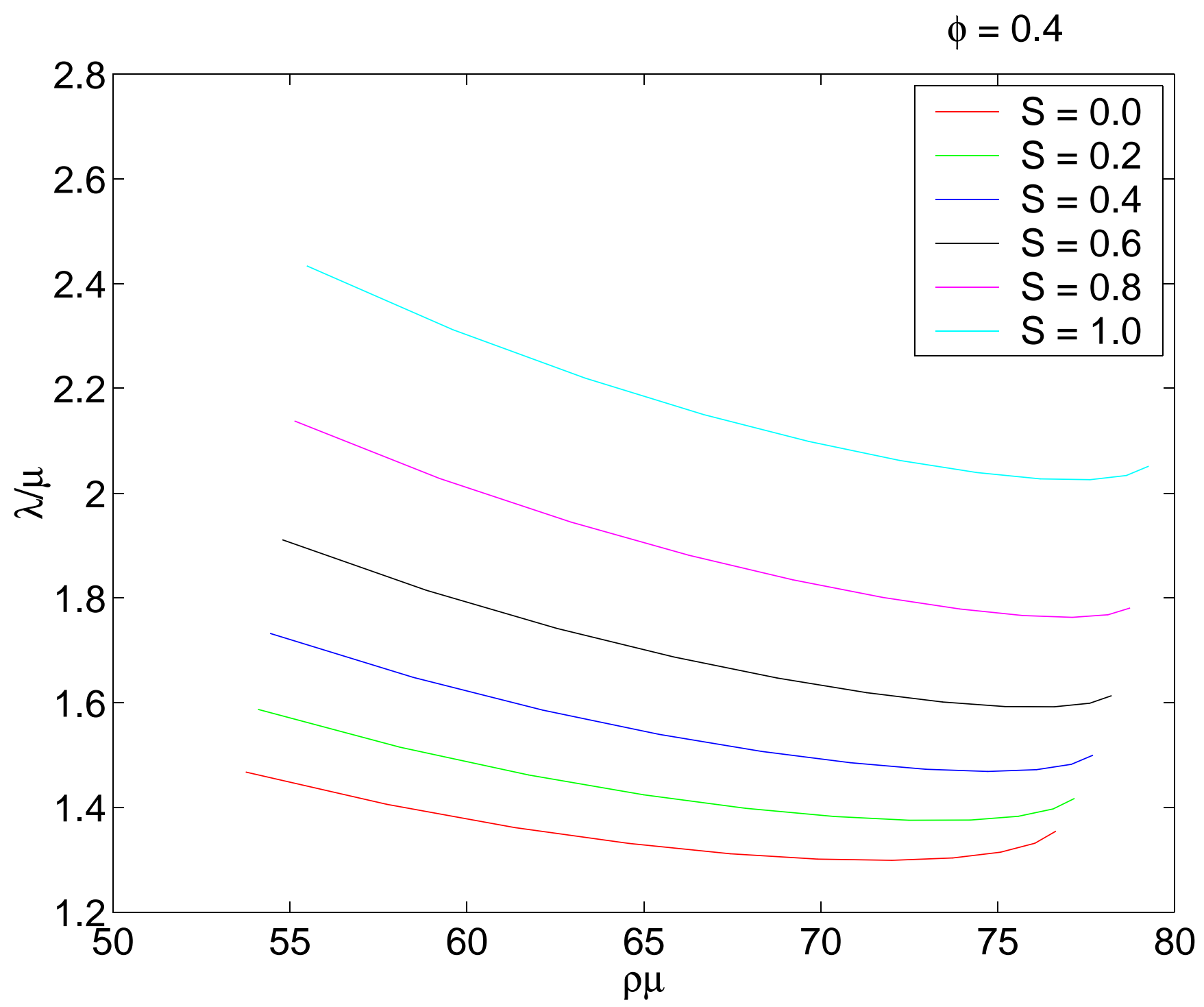

Figure 23: Porosity $\phi=0.4$. Shows the expected inverse correlation with changes of pressure, but some stronger deviations from simple behavior at the lower porosities are observed. Compare the expected behavior of $(\mu$, const $\times 1 / \mu)$. 


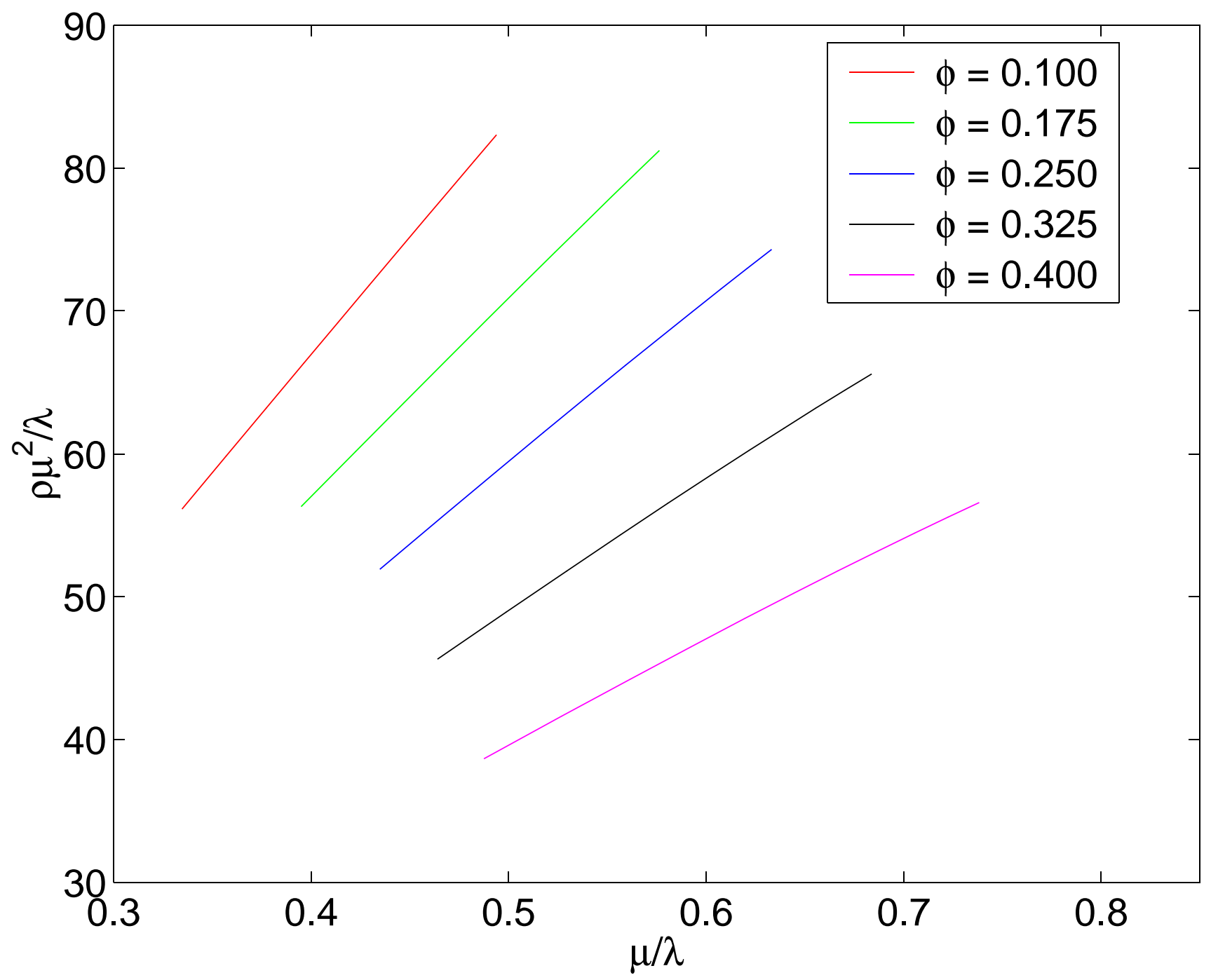

Figure 24: Pressure $=0$ psia. Sorting character of the plot is very clearly observed. Compare the expected behavior of $(\mu / \lambda$, const $\times \mu / \lambda)$. 


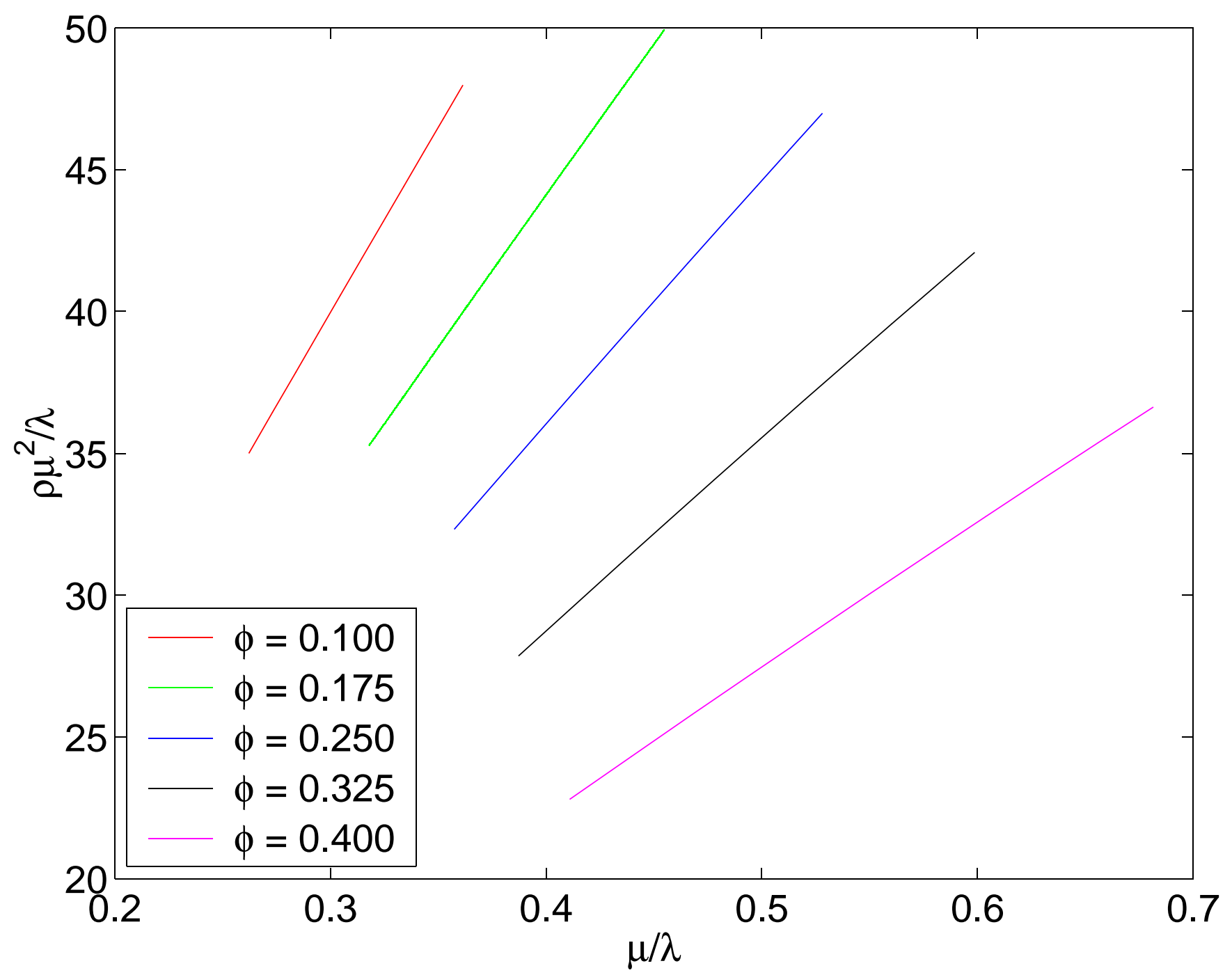

Figure 25: Pressure $=6500$ psia. Sorting character of the plot is very clearly observed. Compare the expeced behavior of $(\mu / \lambda$, const $\times \mu / \lambda)$. 


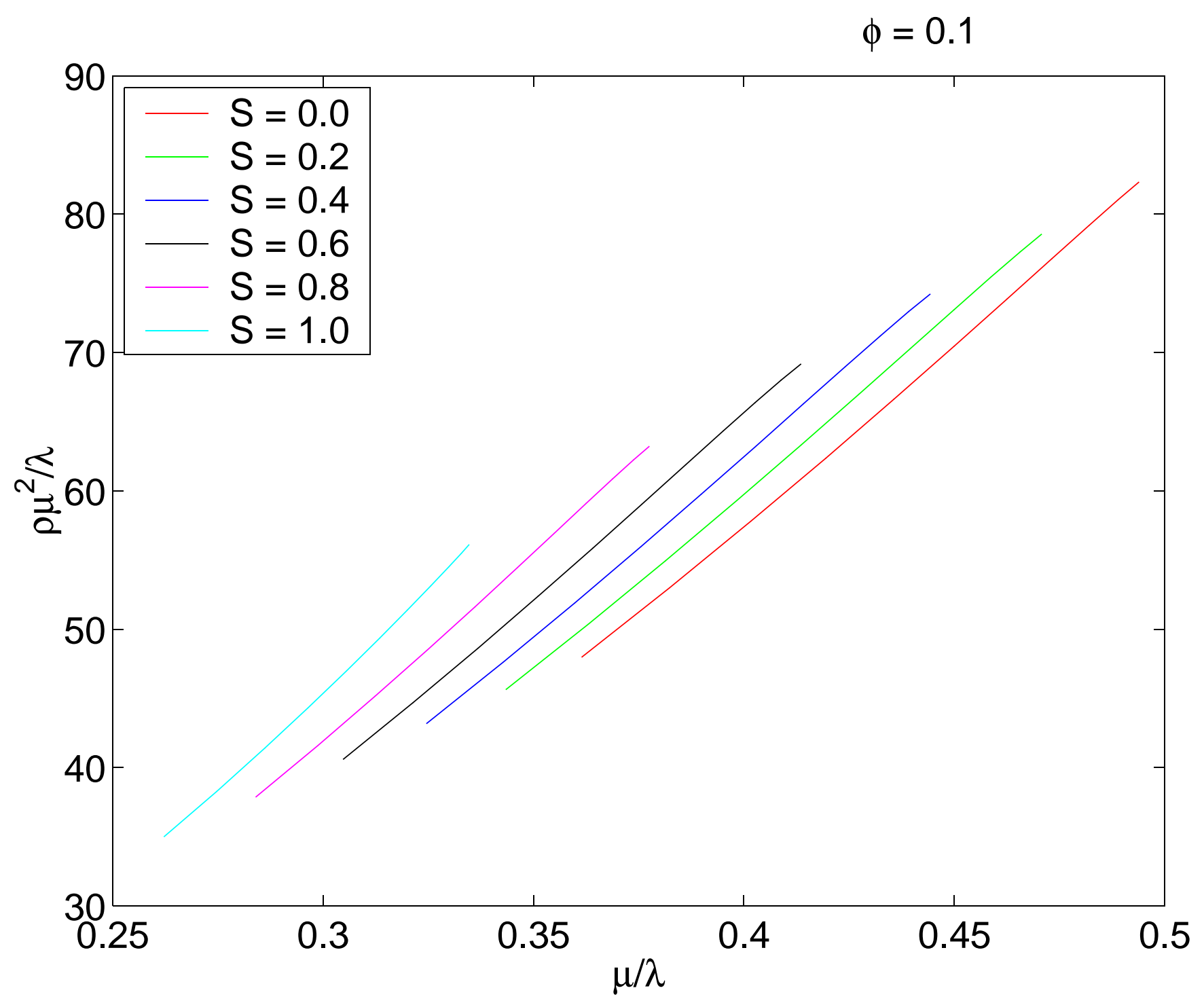

Figure 26: Porosity $\phi=0.1$. Note the weakness of the curvature for all values of saturation. Again, compare $(\mu / \lambda$, const $\times \mu / \lambda)$. 


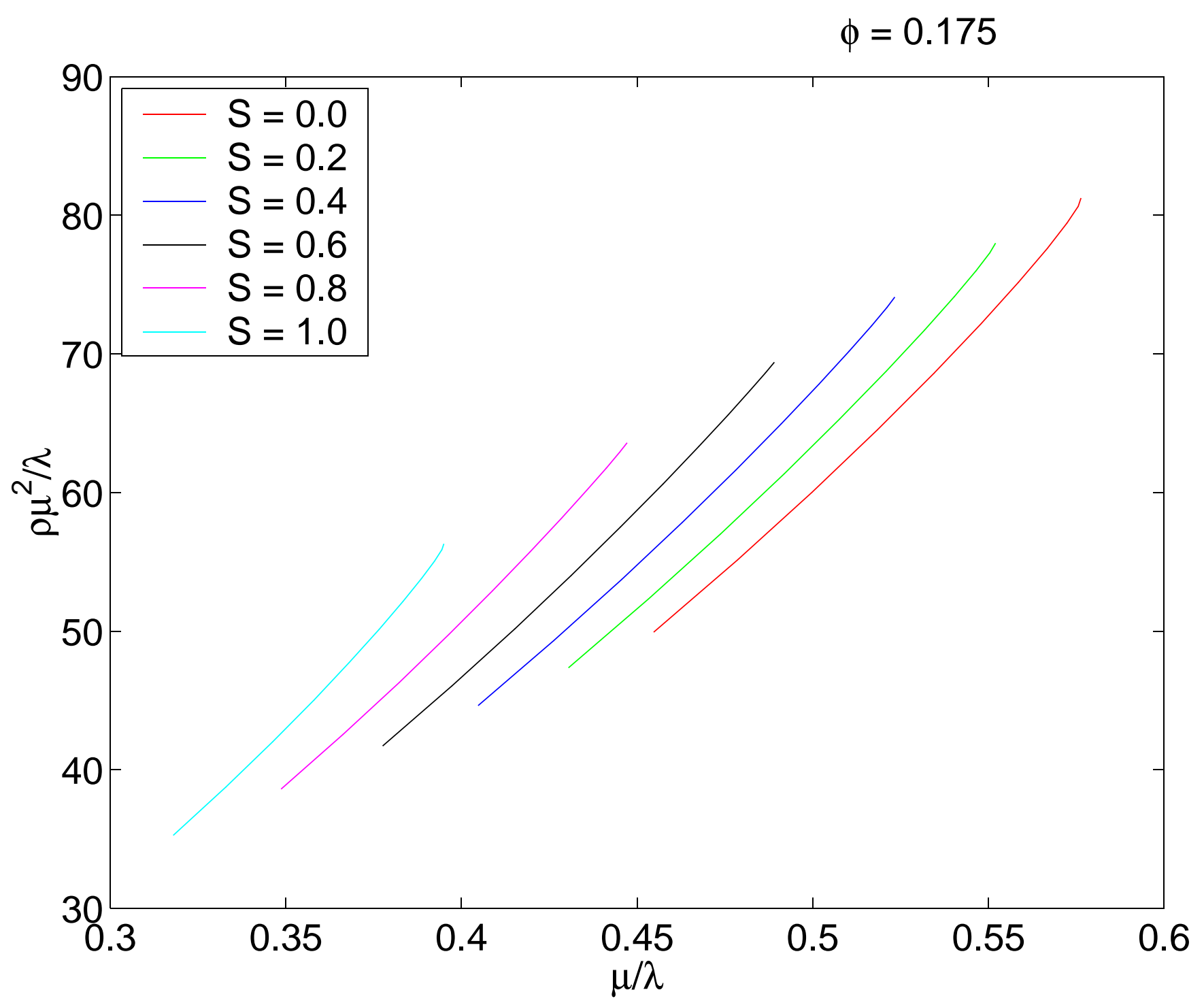

Figure 27: Porosity $\phi=0.175$. Note that some stronger curvature is seen at the smallest values of saturation and the lowest values of the pore pressure. 


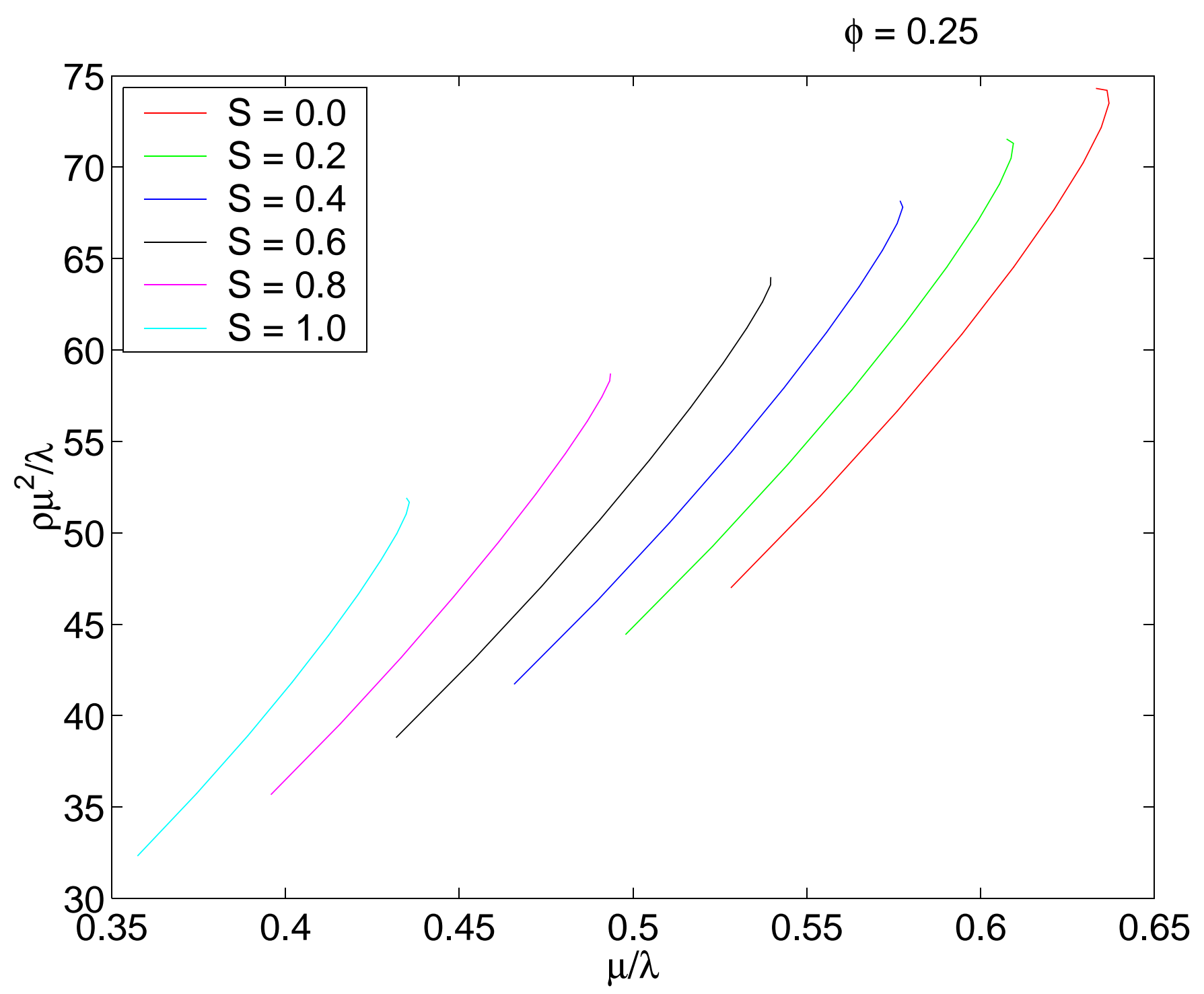

Figure 28: Porosity $\phi=0.25$. Some stronger effects of curvature are starting to show themselves at this value of porosity. 


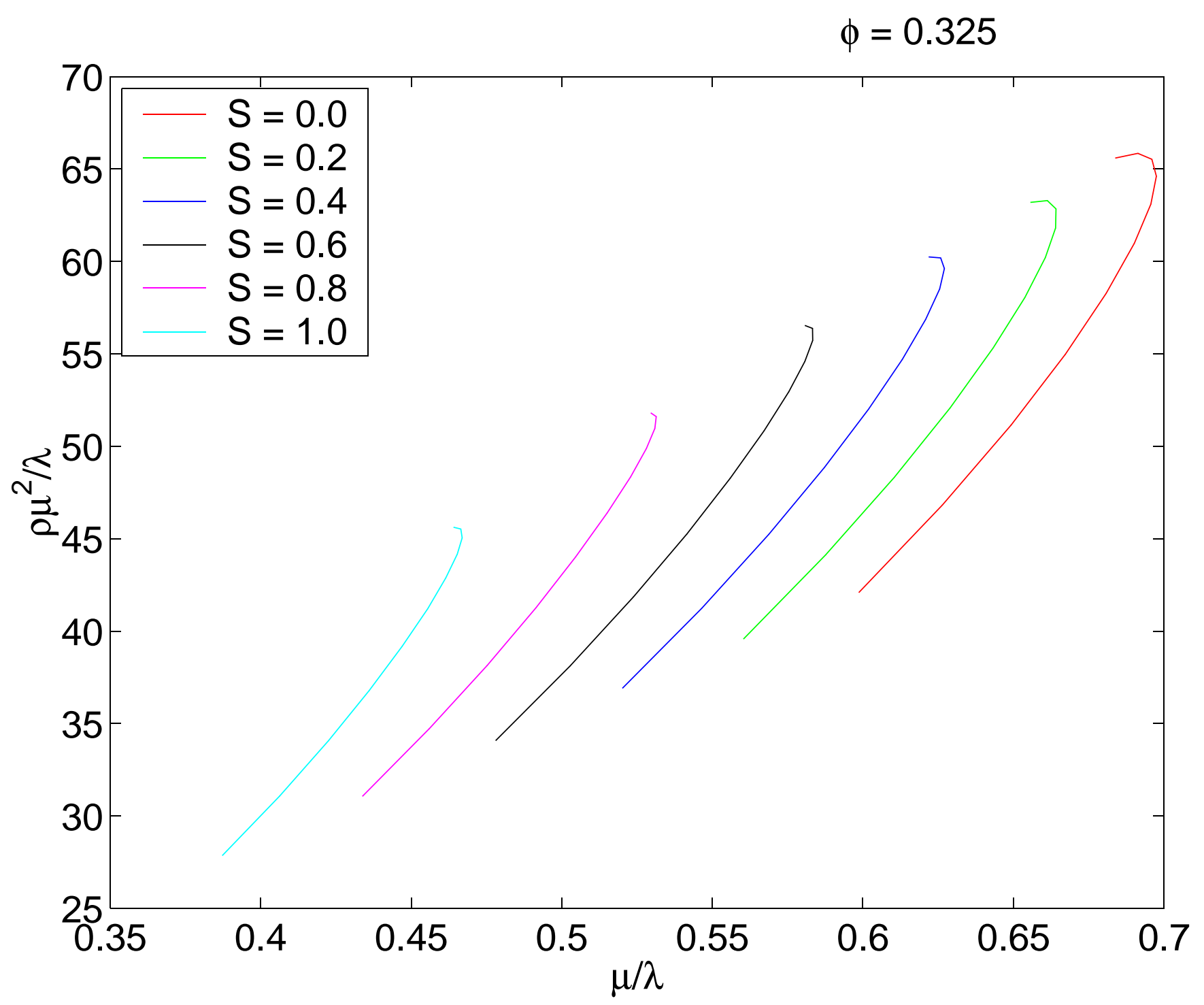

Figure 29: Porosity $\phi=0.325$. Very noticeable curvature is observed for all values of saturation, especially at the lowest values of pressure. 


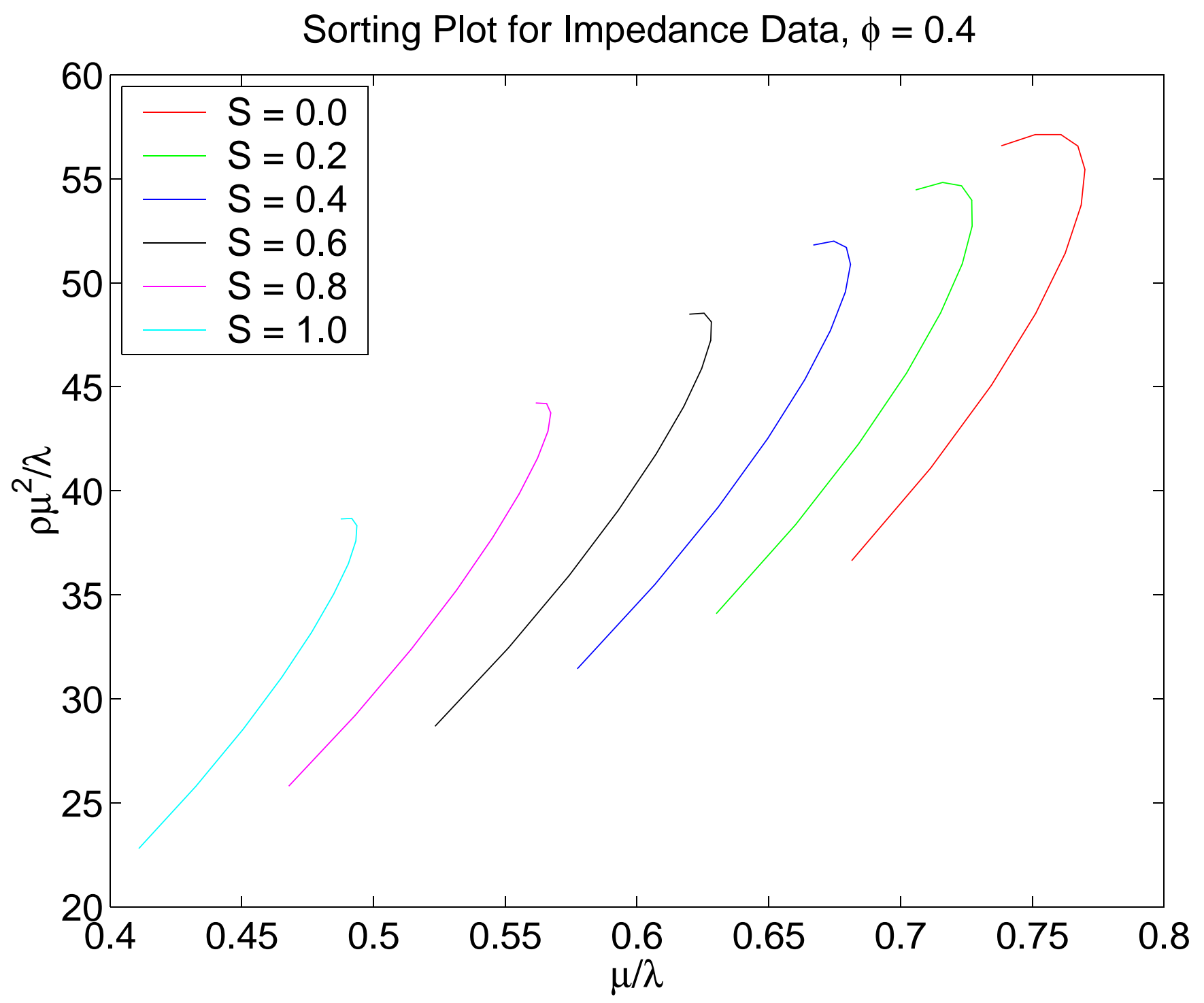

Figure 30: Porosity $\phi=0.4$. Note the strong curvature of these plots for all values of saturation. 


\section{Conclusions}

My main conclusions are that the most appropriate plotting methods for seismic impedance data in order to distinguish saturation, pressure, and saturation from pressure are $(\rho \mu, \lambda / \mu)$ and $\left(\mu / \lambda, \rho \mu^{2} / \lambda\right)$. These are not the only possibilities, but they seem to give the desired results in many situations. Some issues remain concerning the details at high porosities and small pore pressures, and these will need to be addressed in future work.

\section{Acknowledgments}

I thank Steve Cole, David Lumley, Mark Meadows, and Ali Tura, all of 4th Wave Imaging, for supplying me with the synthetic log data used in this report. Work performed under the auspices of the U. S. Department of Energy by the Lawrence Livermore National Laboratory under contract No. W-7405-ENG-48 and supported specifically by the Geosciences Research Program of the DOE Office of Energy Research within the Office of Basic Energy Sciences, Division of Engineering and Geosciences.

\section{References}

[1] J. G. Berryman, P. A. Berge, and B. P. Bonner, "Transformation of seismic velocity data to extract porosity and saturation values for rocks," J. Acoust. Soc. Am. 107, 3018-3027 (2000).

[2] J. G. Berryman, P. A. Berge, and B. P. Bonner, "Estimating rock porosity and fluid saturation using only seismic velocities," Geophysics, submitted and still under review at this time.

[3] F. Gassmann, "Über die Elastizität poroser Medien," Veirteljahrsschrift der Naturforschenden Gesellschaft in Zürich 96, 1-23 (1951). 